\title{
Selection of optimal transmit power in multi-hop underlay cognitive full-duplex relay networks
}

\author{
Poornima S.* (D) and Babu A. V.
}

\begin{abstract}
This paper investigates optimization of underlay multi-hop cognitive full-duplex relay (CogFDR) networks in independent non-identically distributed Rayleigh fading channels. First of all, analytical expressions for the outage probability experienced in the secondary network is formulated, taking into account (i) residual self-interference (RSI) arising due to full-duplex operation, (ii) inter-relay interference (IRI) arising due to frequency re-use, and (iii) interference generated by the primary transmitter on the secondary network. Optimal power allocation (OPA) that either minimizes the end-to-end outage probability or maximizes the end-to-end instantaneous rate is investigated with constraints on total available power in the secondary network and tolerable interference power at the primary receiver. The OPA vector for the outage minimization problem is obtained by solving an equivalent geometric programming problem (GPP) and that for the rate maximization problem is obtained by applying the rate balancing criterion for each hop. Extensive performance evaluations conducted with the help of Monte Carlo simulations show that transmit power optimization can improve the end-to-end rate and outage probability performance of multi-hop CogFDR network in comparison to equal power allocation on average (EPA).
\end{abstract}

Keywords: Full-duplex, Multi-hop, Cognitive, Underlay, Power allocation, Geometric programming

\section{Introduction}

Cognitive radio (CR) networks improve the spectrum utilization efficiency by allowing secondary (unlicensed) users to make use of the primary (licensed) user spectrum without degrading the communication performance of the primary network $[1,2]$. In general, a CR network can operate either in the overlay or in the underlay mode. In the former, the secondary users are allowed to access the spectrum occupied by the primary if the primary user is inactive. In the underlay mode, the secondary users can coexist with the primary user by sharing its frequency band, provided the interference caused by the secondary node transmissions on the primary network, lies within a predefined threshold value. However, this leads to serious constraints on maximum transmit power that the secondary nodes can choose for their operation. To improve the coverage and capacity performance of the secondary

*Correspondence: poornima_p150001ec@nitc.ac.in

Department of Electronics \& Communication Engineering, National Institute of Technology Calicut, Calicut, India network, relay nodes can be deployed to assist the secondary transmitter (ST) in relaying information to the secondary receiver (SR). Such networks that combine the features of cooperative relaying and cognitive radio are known as cognitive relay networks (CRNs) [3].

In CRNs, the relay nodes can operate either in halfduplex (HD) or in full-duplex (FD) mode. In half-duplex relaying (HDR), two orthogonal time or frequency channels are allotted for the relay transmission and reception. Whereas, full-duplex relaying (FDR) allows concurrent reception and retransmission using the same frequency band. Even though FDR leads to two-fold improvement in spectral efficiency, it causes self-interference (SI) because of the coupling between the relay node's transmitter and receiver circuits [4]. Recently several proposals for cancellation of SI have appeared in the literature [5, 6]. However, even with advanced SI cancellation methods, a level of residual self-interference (RSI) remains that significantly deteriorates the performance of FD systems [7-9]. In a FD-based CRN, the relay nodes in the secondary network operate in the FD mode [10]. The CRN based on FD 
would differ in performance in comparison to that of conventional HD network, due to the presence of RSI. This paper considers a cognitive FD relay (CogFDR) network in underlay mode, with a multi-hop secondary network, i.e., the secondary network comprises of a secondary transmitter (ST), a secondary receiver (SR), and $N$ FD relay nodes connecting ST and SR. In underlay CRNs, even though the secondary users are allowed to coexist by sharing the spectrum allotted to the primary, they need to choose the transmission powers such that the interference induced at the primary receiver (PR) does not go beyond the maximum tolerable limit. This significantly affects the outage and rate performance of the secondary user in the CogFDR network. Further, the performance is affected by the amount of RSI as well, which is related to the transmission power of the relay nodes and the power gain of the corresponding channel. The major objective of this paper is to develop a model for finding the secondary users' outage probability in a multi-hop CogFDR system and to investigate transmit power optimization for improving the secondary users' rate and outage probability performance.

\subsection{Related work}

Extensive research has been reported in the literature on the performance evaluation and optimization of CRNs operating in half-duplex mode (i.e., CogHDR networks), e.g., [11-15]. The authors in [11] have considered relay selection for the secondary network in underlay CogHDR with secondary nodes selecting fixed transmit powers for their operation. The outage probability of an underlay decode-and-forward (DF) CogHDR network has been analyzed in [12], while one-dimensional relay location optimization problem has been addressed in [13]. Optimal relay placement in the secondary network of a CogHDR network has been addressed in [14] assuming Nakagami-m fading channels, while the work in [15] has considered transmit power optimization to maximize the achievable ergodic capacity of CogHDR network. In addition to these, many authors have attempted to analyze and improve the performance of wireless networks, see, e.g., [16-19] and references therein. Resource allocation is very crucial in wireless systems. The authors in [16] have considered joint optimization of computation and communication power in multi-user massive MIMO system, which improves the energy efficiency. In [17], the authors have studied the performance of a cooperative multi-relay system where the relays harvest energy from RF signals of the source. The authors in [18] have analyzed the performance of a dual-hop FD system with multiple relays, which provide spectral efficiency and diversity gains respectively. In [19], the authors have proposed a virtual FD relaying scheme for a cooperative multi-path relay channel (MPRC) with multiple half-duplex relays.
Recently, the performance analysis and optimization of dual-hop CogFDR network has been carried out extensively by many researchers [20-29]. In [20], the authors have presented an analytical model to evaluate the outage probability of secondary user in dual-hop CogFDR network by considering a joint decoding scheme at the SR in which the signals arriving at the SR from ST and the relay are utilized together for the decoding purpose. Two-way FD relay spectrum sharing protocol for a dualhop CogFDR network has been proposed in [21], and the outage probability of the network has been evaluated under the proposed protocol. The outage performance of both primary and secondary users in a CogFDR network has been analyzed in [22]. Optimal power allocation algorithms for amplify-and-forward (AF)-based CogFDR network has been analyzed in [23] assuming Rayleigh fading. Two relay selection schemes, i.e., partial relay selection and optimal relay selection, have been proposed in [24], and the outage performance of CogFDR network has been carried out assuming the availability of multiple relay nodes. Joint power control and relay selection algorithms that maximize the transmission rate of the secondary network has been addressed in [25] for a dual-hop CogFDR framework. In [26], the authors have investigated transmit power optimization problem in the context of dual-hop CogFDR network. In [27], the authors have proposed an analytical model for evaluating the outage probability of a dual-hop CogFDR network by considering various locations for the relay node, while neglecting the impact of direct transmission link from ST to SR. In [28], the authors have derived a closed form expression for the outage probability of an OFDM-based CRN with relay selection considering a dual-hop secondary network. In [29], authors have considered optimal power allocation for maximizing the transmission rate in AF-based dual-hop CogFDR network.

In addition to the above papers, a few authors have analyzed the performance of multi-hop CRN as well, i.e., a CRN with multi-hop secondary network [30-35]. In [30], the authors have analyzed optimal power allocation that maximizes the end-to-end throughput for a CRN with multi-hop secondary network; however, RSI is ignored by assuming the intermediate relay nodes to operate in half-duplex mode. In [31, 32], the outage probability of a DF multi-hop half-duplex secondary network with power beacon-assisted energy harvesting based is analyzed. In [33], the authors have analyzed the end-to-end outage probability of a cluster-based multi-hop CRN. The authors of [34] have analyzed the throughput and endto-end outage performance of multi-hop CRN, where the secondary relay nodes harvest energy from PU and preceding secondary relay nodes. In [35], the authors have analyzed the impact of channel information imperfection on the outage performance of multihop CRNs. However, 
all the above mentioned papers [30-35] consider the relay nodes to use HDR while the focus of the current work is on multi-hop CogFDR networks. Even though FDR has been employed in the secondary network of CRNs [20-29], majority of them consider dual-hop secondary network for their analysis of outage and other performance metrics. Finding the transmit power allocation that optimizes the performance of secondary network in multi-hop CogFDR network remains as an open problem.

\subsection{Major objectives and contributions}

In this paper, we focus on the outage probability and instantaneous transmission rate analysis of a multi-hop CogFDR/HDR network. We develop an analytical model for finding the outage probability of the secondary user considering the effects of both RSI and inter-relay interference (IRI), i.e., interference caused by simultaneous transmissions from neighboring nodes that operate in the same frequency band. The interference generated by the primary transmitter on the secondary network is also taken into account for the analysis. We then investigate optimal power allocation for the secondary nodes that either minimizes the end-to-end outage probability or maximizes the instantaneous rate subject to two constraints: (i) constraint imposed on the secondary node's transmit power by the tolerable interference power threshold at PR and (ii) constraint on total transmit power available in the secondary network. We make an extensive study on the end-to-end rate and outage performance of the CogFDR network under optimal power allocation (OPA). For comparison, we report the results for CogHDR network as well. Numerical and simulation results establish that OPA can lead to significant improvement in end-toend rate and outage probability performance of the secondary network in CogFDR system. The analytical results are validated by simulation results.

Remainder of the paper is arranged as follows. Section 2 describes the methods used in this work. Section 3 gives a detailed description of the system model for the underlay CogFDR network. In Section 4, the derivation of the outage probabilities are given. Section 5 describes the transmit power optimization by ignoring the effect of PT, while Section 6 considers the impact of PT for OPA determination. The simulation and analytical results are discussed in Section 7. Finally, the paper is concluded in Section 8.

\section{Methods}

In this paper, we consider a multi-hop underlay cognitive relay network with $N$ DF relays placed between the secondary transmitter and receiver. The outage probability of the secondary network is derived considering the effect of RSI, IRI, and interference from the primary network. The outage probability minimization problem is formulated as a geometric programming problem. The transmit power allocation which maximizes end-to-end rate is determined by equating the rates over each hop. Monte Carlo simulations are done to validate the analytical results.

\section{System model}

The multi-hop CogFDR network that operates in the underlay mode is shown in Fig. 1. Here, the secondary network consists of a secondary transmitter $\left(F_{0}\right)$, secondary

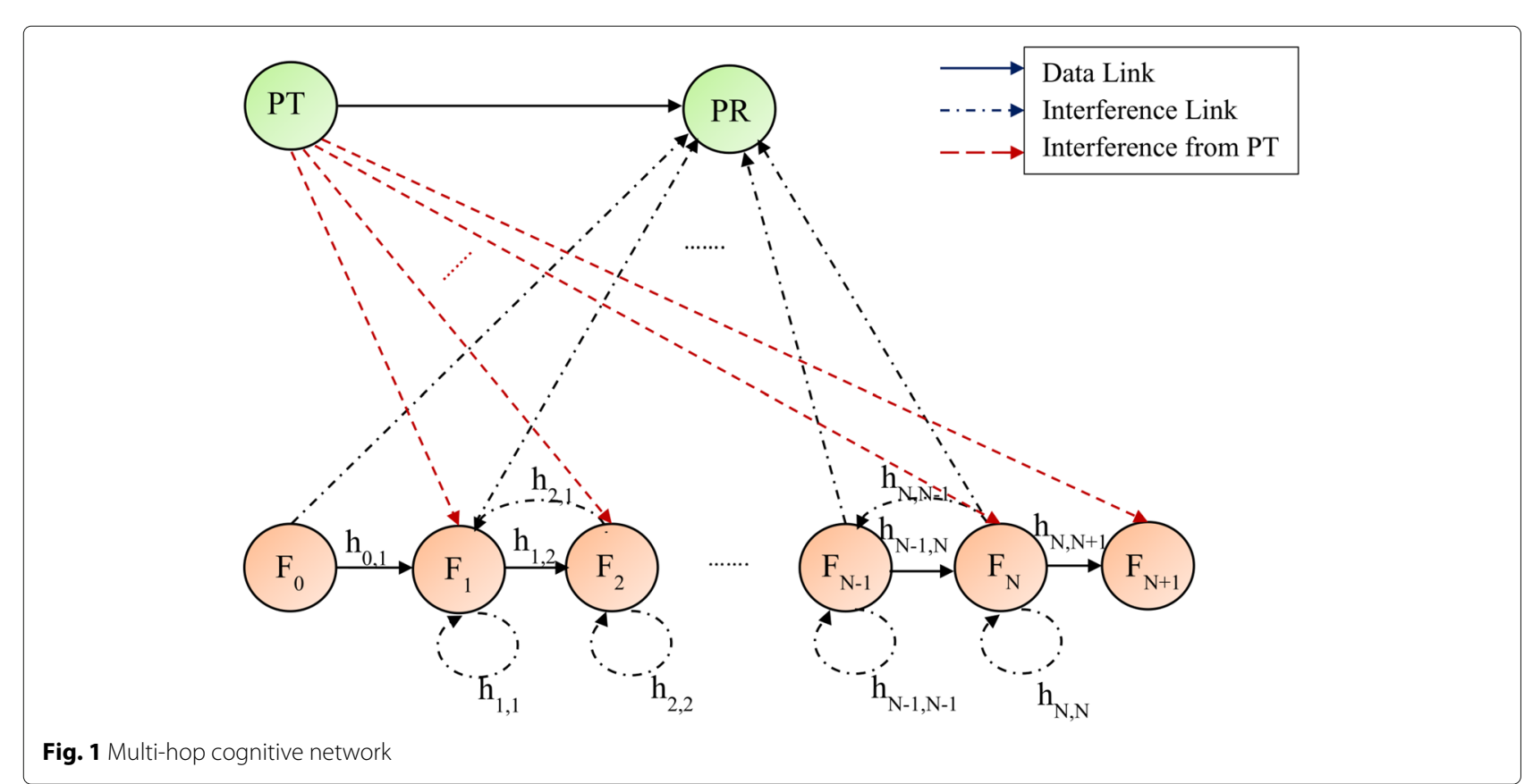


receiver $\left(F_{N+1}\right)$, and $N$ FD relay nodes $\left(F_{1}, \ldots, F_{N}\right)$ that use DF relaying strategy. The primary network consists of a primary transmitter (PT) and a primary receiver (PR). Since the relay nodes in the secondary network operates in FD mode, they suffer from RSI. To further improve the spectral efficiency, multi-hop relay networks employs frequency re-use in which adjacent nodes operate in the same frequency band. Due to simultaneous transmission and reception at the relay nodes, all the receiving nodes in the network suffer from inter-relay interference (IRI) as well. Let $u_{j}$ and $v_{j}$ denote the signals transmitted and received by the relay node $F_{j}$ respectively. Now, $v_{j}$ is composed of the desired signal from $F_{j-1}$; the RSI factor at $F_{j}$; and the IRI components arising due to simultaneous transmissions from the remaining relay nodes $F_{i}, i=0,1, \ldots N, \forall i \neq j-1, j$. The path loss exponent is assumed to be relatively higher for the links in the network. The path loss exponent can be very large in certain scenarios such as obstructed in-building environment. Since the signal is attenuated significantly as the path loss exponent becomes larger, we assume that the IRI at the relay node $F_{j}$ consists of interference from one hop neighbor alone (i.e., from relay node $F_{j+1}$ alone), i.e., the IRI components arriving at the relay $F_{j}$ from all the other links are assumed to be negligible as shown in Fig. 1. Further, we assume that $F_{j}$ does not employ any diversity technique; thus, the signal that arrives at $F_{j}$ from $F_{j+1}$ is treated as interference All the links in the network undergo independent non-identically distributed frequency flat Rayleigh fading.

A block fading model has been assumed where fading is assumed to be constant over one block but changes independently from one block to another [36]. Furthermore, we assume that all the FD relays employ two directional antennas, solely for transmission and reception, respectively. Directional antennas isolate the transmitter and receiver circuits of each FDR node, so that the effect of loop interference can be reduced. Further, directional antennas can reduce the line-of-sight (LOS) components of SI as well [7]. As reported in many papers, e.g., [4-10, 20-27], we model the RSI channel of every relay node as a Rayleigh fading channel. Let the channel gain between relay nodes $F_{i}$ and $F_{j}$ be $h_{i, j}, i, j \in\{0,1, \ldots . N+1\}$, and let $h_{j, j}$ be the channel coefficient of the RSI channel at the relay node $F_{j}, j=\{1,2, \ldots . N\}$. With Rayleigh fading assumption, $\left|h_{i, j}\right|^{2}$ are exponential, having mean values $\lambda_{i, j}$. Furthermore, we consider $h_{i, P R}$ and $h_{P T, j}$ to represent the channel coefficient corresponding to the links connecting secondary nodes $F_{i}, i=\{0,1, \ldots N\}$ to PR and PT to $F_{j}, j=\{1, \ldots N+1\}$, respectively. With Rayleigh fading assumption, the power gains $\left|h_{P T, j}\right|^{2}$ and $\left|h_{i, P R}\right|^{2}$ are exponential with mean values $\lambda_{P T, j}$ and $\lambda_{i, P R}$, respectively. The average channel power gains $\lambda_{i, j}, i, j \in\{0,1, \ldots . N\}$ are related to the length of the corresponding links as $\lambda_{i, j}=G D_{i, j}^{-n}$ where $n$ is the path loss exponent; $D_{i, j}$ is the distance between $F_{i}$ and $F_{j}$, and $G$ is the propagation constant [36]. The mean channel gains for the link connecting PT to a node in the secondary network is given by $\lambda_{P T, j}=G D_{P T, j}^{-n}, j=\{1,2, \ldots N+1\}$ and that for the link from secondary nodes to $\mathrm{PR}$ is given by $\lambda_{i, P R}=G D_{i, P R}^{-n}, i=$ $\{0,1, \ldots N\}$, where $D_{P T, j}$ and $D_{i, P R}$ denotes the respective distances from $\mathrm{PT}$ to $F_{j}$ and from $F_{i}$ to $\mathrm{PR}$, respectively. The mean RSI, denoted by $\lambda_{j, j}, j=\{1,2, \ldots N\}$, is assumed as equal for all the FD relays. Some reduction in IRI is also attained by the usage of antennas that are directional in nature, at the FDR nodes. Hence, the amount of IRI at $F_{j}$ from its successor node $F_{j+1}$ can be assumed to be comparatively less. Accordingly, the mean IRI at $F_{j}$ is modeled as $\lambda_{j+1, j}=\delta G D_{j+1, j}^{-n}$, where $0<\delta<1$ denotes the fraction of IRI reduction due to the use of directional antennas. Apart from this, we model the noise as white Gaussian noise with zero mean and variance $N_{0}$. The received signal at relay node $F_{j}$ during a given time instance is given by

$$
v_{j}=h_{j-1, j} u_{j-1}+h_{j j} u_{j}+h_{j+1, j} u_{j+1}+n_{j}
$$

where $n_{j}$ denotes the additive white Gaussian noise at $F_{j}$. In addition to this, $u_{j-1}, u_{j}$, and $u_{j+1}$ are the signals transmitted by $F_{j-1}, F_{j}$, and $F_{j+1}$, respectively, during the specified time instance.

\section{Derivation of outage probability of the secondary user}

In this section, we derive analytical expressions for the secondary user's outage probability for the following cases: (i) by ignoring interference from PT and (ii) by considering the effect of interference from PT. We derive the outage probability expression for the CogHDR network as well.

\subsection{Outage probability derivation ignoring interference from primary transmitter}

In this section, we assume the PT to be located far away from the secondary network so that primary transmission does not cause any interference to the secondary network. Here, we derive the outage probability expressions for the multi-hop CogFDR and CogHDR networks.

\subsubsection{Multi-hop CogFDR network}

Define $P_{i}, i=\{0,1, \ldots N\}$ as the transmission power vector for the secondary nodes of the CogFDR network in Fig. 1. Further, let $\Gamma_{j, \mathrm{FDR}}^{(0)}$ be the signal-to-interference plus noise ratio (SINR) at the relay node $F_{j}, j=\{1,2, \ldots N-1\}$, which is computed as follows:

$$
\Gamma_{j, \mathrm{FDR}}^{(0)}=\frac{P_{j-1}\left|h_{j-1, j}\right|^{2}}{N_{0}+P_{j}\left|h_{j, j}\right|^{2}+P_{j+1}\left|h_{j+1, j}\right|^{2}}
$$


Furthermore, the SINR at the relay nodes $F_{N}$ and $F_{N+1}$ (i.e., $\Gamma_{N, \mathrm{FDR}}^{(0)}$ and $\Gamma_{N+1, \mathrm{FDR}}^{(0)}$, respectively) are determined as follows:

$$
\begin{aligned}
\Gamma_{N, \mathrm{FDR}}^{(0)} & =\frac{P_{N-1}\left|h_{N-1, N}\right|^{2}}{N_{0}+P_{N}\left|h_{N, N}\right|^{2}} \\
\Gamma_{N+1, \mathrm{FDR}}^{(0)} & =\frac{P_{N}\left|h_{N, N+1}\right|^{2}}{N_{0}}
\end{aligned}
$$

Define $X_{i, j}=P_{i}\left|h_{i, j}\right|^{2} / N_{0}$. Accordingly, $\Gamma_{j, \mathrm{FDR}}^{(0)}, j=$ $\{1,2, \ldots N-1\}$ can be rewritten as,

$$
\Gamma_{j, \mathrm{FDR}}^{(0)}=\frac{X_{j-1, j}}{1+X_{j, j}+X_{j+1, j}}
$$

The outage probability of a multi-hop CogFDR network with DF relaying is the probability for at least one of the links along the multi-hop path to experience outage. Assuming the fading over the links to be independent, the probability of outage is computed by,

$$
P_{\mathrm{out}, \mathrm{FDR}}^{(0)}=1-\prod_{j=1}^{N+1} \operatorname{Pr}\left(\Gamma_{j, \mathrm{FDR}}^{(0)} \geq \Gamma_{T}\right)
$$

where $\Gamma_{T}$ is the threshold SINR required for acceptable performance over link $j, j=1,2, \ldots N+1$. Let $r$ (bits/sec/Hz) be the target data rate for communication over link $j$ connecting nodes $F_{j-1}$ and $F_{j}$. Now, $r$ and $\Gamma_{T}$ are related as follows: $\Gamma_{T}=2^{r}-1$ [7]. Now, for $j=1,2, \ldots N-1$, we find the probability $\operatorname{Pr}\left(\Gamma_{j, \mathrm{FDR}}^{(0)} \geq \Gamma_{T}\right)$ as follows:

$$
\operatorname{Pr}\left(\Gamma_{j, \mathrm{FDR}}^{(0)} \geq \Gamma_{T}\right)=\operatorname{Pr}\left(\frac{X_{j-1, j}}{1+X_{j, j}+X_{j+1, j}}>\Gamma_{T}\right)
$$

Let $Z_{j}=X_{j, j}+X_{j+1, j}$. Since $\left|h_{i, j}\right|^{2}$ is exponential with mean value $\lambda_{i, j}, X_{i, j}$ is also exponential with the mean value $\beta_{i, j}=\frac{\lambda_{i, j} P_{i}}{N_{0}}$. The probability density function (PDF) of $X_{i, j}$ is given by,

$$
f_{X_{i, j}}(x)=\frac{1}{\beta_{i, j}} \exp \left(-\frac{x}{\beta_{i, j}}\right), x>0
$$

Since $h_{i, j}$ 's are assumed to be independent random variables, the PDF of $Z_{j}$ can be determined as the convolution of the PDFs of $X_{j, j}$ and $X_{j+1, j}$ and is given by,

$$
\begin{aligned}
f_{Z_{j}}(z) & =\int_{0}^{z} f_{X_{j, j}}(x) f_{X_{j+1, j}}(z-x) d x \\
& =\int_{0}^{z} \frac{1}{\beta_{j, j}} \exp \left(-\frac{x}{\beta_{j, j}}\right) \frac{1}{\beta_{j+1, j}} \exp \left(-\frac{(z-x)}{\beta_{j+1, j}}\right) d x \\
& =\frac{1}{\left(\beta_{j+1, j}-\beta j, j\right)}\left[\exp \left(-\frac{z}{\beta_{j+1, j}}\right)-\exp \left(-\frac{z}{\beta_{j, j}}\right)\right], z>0
\end{aligned}
$$

Utilizing (7) and (8), $\operatorname{Pr}\left(\Gamma_{j, \mathrm{FDR}}^{(0)} \geq \Gamma_{T}\right), j=1,2, \ldots N-1$ can be determined as follows (details given in Appendix A):

$$
\begin{aligned}
\operatorname{Pr}\left(\Gamma_{j, \mathrm{FDR}}^{(0)} \geq \Gamma_{T}\right) & =\operatorname{Pr}\left(\frac{X_{j-1, j}}{1+Z_{j}} \geq \Gamma_{T}\right) \\
& =\frac{e^{-\frac{\Gamma_{T}}{\beta_{j-1, j}}}}{\left(1+\frac{\Gamma_{T} \beta_{j+1, j}}{\beta_{j-1, j}}\right)\left(1+\frac{\Gamma_{T} \beta_{j, j}}{\beta_{j-1, j}}\right)}
\end{aligned}
$$

The probability $\operatorname{Pr}\left(\Gamma_{N, \mathrm{FDR}}^{(0)} \geq \Gamma_{T}\right)$ is computed as follows:

$$
\begin{aligned}
\operatorname{Pr}\left(\Gamma_{N, \mathrm{FDR}}^{(0)} \geq \Gamma_{T}\right) & =\operatorname{Pr}\left(\frac{P_{N-1}\left|h_{N-1, N}\right|^{2}}{N_{0}+P_{N}\left|h_{N, N}\right|^{2}} \geq \Gamma_{T}\right) \\
& =\operatorname{Pr}\left(\frac{X_{N-1, N}}{1+X_{N, N}} \geq \Gamma_{T}\right) \\
& =\int_{x=0}^{\infty} e^{-\frac{\Gamma_{T}(1+x)}{\beta_{N-1, N}}} f_{X_{N, N}}(x) d x \\
& =\int_{x=0}^{\infty} e^{-\frac{\Gamma_{T}(1+x)}{\beta_{N-1, N}}} \frac{1}{\beta_{N, N}} \exp \left(\frac{-x}{\beta_{N, N}}\right) d x \\
& =\frac{e^{\frac{-\Gamma_{T}}{\beta_{N-1, N}}}}{1+\frac{\Gamma_{T} \beta_{N, N}}{\beta_{N-1, N}}}
\end{aligned}
$$

The probability $\operatorname{Pr}\left(\Gamma_{N+1, \mathrm{FDR}}^{(0)} \geq \Gamma_{T}\right)$ is computed as follows:

$$
\begin{aligned}
\operatorname{Pr}\left(\Gamma_{N+1, \mathrm{FDR}}^{(0)} \geq \Gamma_{T}\right) & =\operatorname{Pr}\left(\frac{P_{N}\left|h_{N, N+1}\right|^{2}}{N_{0}} \geq \Gamma_{T}\right) \\
& =1-\left(1-e^{\frac{-\Gamma_{T}}{\beta_{N, N+1}}}\right) \\
& =e^{\frac{-\Gamma_{T}}{\beta_{N, N+1}}}
\end{aligned}
$$

The end-to-end outage probability experienced by a secondary user $P_{\text {out,FDR }}^{(0)}$ is calculated by substituting (9)-(11) in (5). In the underlay mode, since the secondary network is allowed to occupy the spectrum of the primary, we need to ensure that the interference at PR caused by transmissions within the secondary network is below the tolerable threshold level. Recall that outage probability is a performance metric applicable for slow-fading conditions [36], when the channel becomes unusable for certain fraction of time. From (9)-(11), it can be seen that the outage probability is a function of average channel gains. Thus, we assume that transmit powers of secondary source and relays are strictly limited by the average tolerable interference at PR [37]. Let $I_{\text {avg }}$ be the average tolerable 
threshold at PR. Now, $P_{j}, j=\{0,1, \ldots N\}$ have to be chosen such that the following conditions are satisfied:

$$
\begin{gathered}
\sum_{j=0}^{N} P_{j} \lambda_{j, P R} \leq I_{\mathrm{avg}} \\
\sum_{j=0}^{N} P_{j}=P
\end{gathered}
$$

To satisfy the above constraints, the transmit powers for the secondary nodes are chosen as:

$$
P_{j}=\min \left(\frac{P}{N+1}, \frac{I_{\mathrm{avg}}}{(N+1) \lambda_{j, P R}}\right), j=\{0,1, \ldots N\}
$$

Selection of $P_{j}$ according to (13) is known as equal power allocation on average (EPA) [26]. Notice that EPA does not optimize the system performance. In Section 5, we consider OPA for minimizing the outage probability.

\subsubsection{Multi-hop CogHDR network}

We consider a CogHDR network with multi-hop secondary system consisting of $N$ relay nodes $F_{i}, i=$ $1,2, \ldots N$, a source node $F_{0}$ and a destination node $F_{N+1}$ as in the CogFDR case. However, we consider a multi-phase relaying system where $N+1$ non overlapping time slots are allocated for transmission within the secondary network. As a result, the spectral efficiency is degraded significantly; however, the IRI is zero. Further, HDR networks do not suffer from RSI [7]. Accordingly, the received SNR at the relay node $F_{j}$ is given by,

$$
\Gamma_{j, \mathrm{HDR}}^{(0)}=\frac{P_{j-1}\left|h_{j-1, j}\right|^{2}}{N_{0}}, j=1,2, \ldots . N+1
$$

As in the CogFDR case outlined earlier, the outage probability experienced by a secondary user is represented as follows:

$$
P_{\mathrm{out}, \mathrm{HDR}}^{(0)}=1-\prod_{j=1}^{N+1} \operatorname{Pr}\left(\Gamma_{j, \mathrm{HDR}}^{(0)} \geq \gamma_{T}\right)
$$

where $\gamma_{T}$ is the SINR threshold for the CogHDR system. For a fair comparison, we set $\gamma_{T}=2^{r(N+1)}-1$ assuming that each node in the secondary network of CogHDR system consumes $\left(\frac{1}{N+1}\right)$ th fraction of the total resources available [7]. Since $\Gamma_{j, \mathrm{HDR}}^{(0)}=X_{j-1, j}$ which is exponential with the mean value $\beta_{j-1, j}=\frac{\lambda_{j-1, j} P_{j-1}}{N_{0}}, P_{\text {out }, \mathrm{HDR}}^{(0)}$ is represented by the following equation

$$
P_{\mathrm{out}, \mathrm{HDR}}^{(0)}=1-\prod_{j=1}^{N+1} \exp \left(-\gamma_{T} / \beta_{j-1, j}\right)
$$

Since the relay nodes operate in HD mode, $P_{j}, j=$ $\{0,1, \ldots N\}$ have to satisfy the following constraints:

$$
\begin{aligned}
P_{j} \lambda_{j, P R} & \leq I_{\text {avg }}, j=\{0,1, \ldots N\} \\
\sum_{j=0}^{N} P_{j} & =P
\end{aligned}
$$

To satisfy (17), $P_{j}, j=\{0,1, \ldots N\}$ for the CogHDR network is given by,

$$
P_{j}=\min \left(\frac{P}{N+1}, \frac{I_{\text {avg }}}{\lambda_{j, P R}}\right), j=\{0,1, \ldots N\}
$$

\subsection{Outage probability of secondary user under the effect of interference from the primary transmitter}

In this section, we find the outage probability of the secondary network for CogFDR and CogHDR scenarios by taking into account the effect of interference from PT on the secondary network performance. Assume that PT transmits a signal $x_{p}$ to PR with fixed power $P_{P T}$ and data rate $R_{p}$. The secondary nodes re-use this time resource to transmit their signals.

\subsubsection{Multi-hop CogFDR network}

With the primary interference induced on the secondary network, the instantaneous SINR at the node $F_{j}, j=$ $\{1,2, \ldots N-1\}$ can be written as follows:

$$
\Gamma_{j, \mathrm{EDR}}^{(1)}=\frac{P_{j-1}\left|h_{j-1, j}\right|^{2}}{N_{0}+P_{j}\left|h_{j, j}\right|^{2}+P_{j+1}\left|h_{j+1, j}\right|^{2}+P_{P T}\left|h_{P T, j}\right|^{2}}
$$

Similar to Section 4.1.1, we can rewrite (19) as follows:

$$
\Gamma_{j, \mathrm{FDR}}^{(1)}=\frac{X_{j-1, j}}{1+X_{j, j}+X_{j+1, j}+X_{P T, j}}
$$

where $X_{P T, j}=P_{P T}\left|h_{P T, j}\right|^{2} / N_{0}$. The outage probability of the secondary network for this scenario is defined as,

$$
P_{\mathrm{out}, \mathrm{FDR}}^{(1)}=1-\prod_{j=1}^{N+1} \operatorname{Pr}\left(\Gamma_{j, \mathrm{FDR}}^{(1)} \geq \Gamma_{T}\right)
$$

Now $\operatorname{Pr}\left(\Gamma_{j, \mathrm{FDR}}^{(1)} \geq \Gamma_{T}\right), j=\{1,2, \ldots N-1\}$ are determined as follows:

$\operatorname{Pr}\left(\Gamma_{j, \mathrm{FDR}}^{(1)} \geq \Gamma_{T}\right)=\operatorname{Pr}\left(\frac{X_{j-1, j}}{1+X_{j, j}+X_{j+1, j}+X_{P T, j}} \geq \Gamma_{T}\right)$

Assuming independent, non-identically distributed Rayleigh fading links, (22) can be evaluated as follows (details given in Appendix B):

$$
\operatorname{Pr}\left(\Gamma_{j, \mathrm{FDR}}^{(1)} \geq \Gamma_{T}\right)=e^{-\frac{\Gamma_{T}}{\beta_{j-1, j}}}\left(1+\frac{\Gamma_{T} \beta}{\beta_{j-1, j}}\right)^{-m}
$$


where $m$ and $\beta$ are defined as:

$$
\begin{gathered}
m=\frac{\left(\beta_{j, j}+\beta_{j+1, j}+\beta_{P T, j}\right)^{2}}{\beta_{j, j}^{2}+\beta_{j+1, j}^{2}+\beta_{P T, j}^{2}} \\
\beta=\frac{\beta_{j, j}^{2}+\beta_{j+1, j}^{2}+\beta_{P T, j}^{2}}{\beta_{j, j}+\beta_{j+1, j}+\beta_{P T, j}}
\end{gathered}
$$

Similar to (10), $\operatorname{Pr}\left(\Gamma_{N, \mathrm{FDR}}^{(1)} \geq \Gamma_{T}\right)$ is given by,

$$
\begin{aligned}
\operatorname{Pr}\left(\Gamma_{N, \mathrm{FDR}}^{(1)} \geq \Gamma_{T}\right) & =\operatorname{Pr}\left(\frac{P_{N-1}\left|h_{N-1, N}\right|^{2}}{N_{0}+P_{N}\left|h_{N, N}\right|^{2}+P_{P T}\left|h_{P T, N}\right|^{2}} \geq \Gamma_{T}\right) \\
& =\operatorname{Pr}\left(\frac{X_{N-1, N}}{1+X_{N, N}+X_{P T, N}} \geq \Gamma_{T}\right) \\
& =\frac{e^{-\frac{\Gamma_{T}}{\beta_{N-1, N}}}}{\left(1+\frac{\Gamma_{T} \beta_{P T, N}}{\beta_{N-1, N}}\right)\left(1+\frac{\Gamma_{T} \beta_{N, N}}{\beta_{N-1, N}}\right)}
\end{aligned}
$$

Similarly $\operatorname{Pr}\left(\Gamma_{N+1, \mathrm{FDR}}^{(1)} \geq \Gamma_{T}\right)$ is given by,

$$
\begin{aligned}
\operatorname{Pr}\left(\Gamma_{N+1, \mathrm{FDR}}^{(1)} \geq \Gamma_{T}\right) & =\operatorname{Pr}\left(\frac{P_{N}\left|h_{N, N+1}\right|^{2}}{N_{0}+P_{P T}\left|h_{P T, N+1}\right|^{2}} \geq \Gamma_{T}\right) \\
& =\operatorname{Pr}\left(\frac{X_{N, N+1}}{1+X_{P T, N+1}} \geq \Gamma_{T}\right) \\
& =\frac{e^{\frac{-\Gamma_{T}}{\beta_{N, N+1}}}}{1+\frac{\Gamma_{T} \beta_{P T, N+1}}{\beta_{N, N+1}}}
\end{aligned}
$$

Now, $P_{\text {out,FDR }}^{(1)}$ is determined by substituting (23a)-(25) in (21).

\subsubsection{Multi-hop CogHDR network}

Considering the effect of interference from PT, the SINR at the relay node $F_{j}, \Gamma_{j, H D R}^{(1)}$ is given by,

$$
\begin{aligned}
\Gamma_{j, \mathrm{HDR}}^{(1)} & =\frac{P_{j-1}\left|h_{j-1, j}\right|^{2}}{N_{0}+P_{P T}\left|h_{P T, j}\right|^{2}}, j=1,2, \ldots . N+1 \\
& =\frac{X_{j-1, j}}{1+X_{P T, j}}
\end{aligned}
$$

The outage probability $P_{\text {out }, \mathrm{HDR}}^{(1)}$ is then computed as follows (details given in Appendix C):

$$
\begin{aligned}
P_{\mathrm{out}, \mathrm{HDR}}^{(1)} & =1-\prod_{j=1}^{N+1} \operatorname{Pr}\left(\Gamma_{j, \mathrm{HDR}}^{(1)} \geq \gamma_{T}\right) \\
& =1-\prod_{j=1}^{N+1} \operatorname{Pr}\left(\frac{X_{j-1, j}}{1+X_{P T, j}} \geq \gamma_{T}\right) \\
& =1-\prod_{j=1}^{N+1} \frac{e^{-\frac{\gamma_{T}}{\beta_{j-1, j}}}}{\left(1+\frac{\gamma_{T} \beta_{P T, j}}{\beta_{j-1, j}}\right)}
\end{aligned}
$$

\section{Optimal power allocation (OPA) for the secondary nodes in CogFDR network ignoring primary interference}

In this section, we consider optimizing the performance of CogFDR secondary network ignoring the influence of the interference from the primary network. We consider two optimization problems for the secondary network: (i) determine the OPA to minimize the end-to-end outage probability and (ii) determine the OPA to maximize the end-to-end instantaneous rate.

\subsection{Outage probability minimization in multi-hop CogFDR network}

Here, we determine the OPA for the secondary network which minimizes the outage probability of a CogFDR system with average interference constraint at PR. The optimization problem is formulated as follows:

$$
\begin{aligned}
\underset{\substack{P_{j} \\
j=0,1, \ldots N}}{\operatorname{Min}} & P_{\mathrm{out}, \mathrm{FDR}}^{(0)} \\
\text { s.t. } & \sum_{j=0}^{N} P_{j} \leq P \\
& \sum_{j=0}^{N} P_{j} \lambda_{j, P R} \leq I_{\mathrm{avg}} \\
& P_{j}>0, j=0,1,2, \ldots N
\end{aligned}
$$

Here, (28b) is the sum power constraint and (28c) represents the average interference constraint at PR, which limits the average interference caused by the nodes in the secondary network at PR. Let us define $\mathbf{P}=\left[P_{j}\right], j=$ $\{0,1, . . N\}$. Since minimizing $P_{\text {out,FDR }}^{(0)}$ is equal to minimizing the function $F(\mathbf{P})=-\ln \left(1-P_{\text {out,FDR }}^{(0)}\right)$, we can reformulate the optimization problem in (28) as follows:

$$
\begin{array}{cl}
\underset{\substack{P_{j} \\
j=0,1, \ldots N}}{\operatorname{Min}} & F(\mathbf{P})=-\ln \left(1-P_{\text {out,FDR }}^{(0)}\right) \\
\text { s.t. } & \sum_{j=0}^{N} P_{j} \leq P \\
& \sum_{j=0}^{N} P_{j} \lambda_{j, P R} \leq I_{\text {avg }} \\
& P_{j}>0, j=0,1,2, \ldots N
\end{array}
$$

The objective function in (29a) can be simplified as follows: 


$$
\begin{aligned}
F(\mathbf{P}) & =-\ln \left(\prod_{j=1}^{N+1} \operatorname{Pr}\left(\Gamma_{j, \mathrm{FDR}}^{(0)} \geq \Gamma_{T}\right)\right) \\
& =\sum_{j=1}^{N+1}\left(\frac{\Gamma_{T}}{\beta_{j-1, j}}+\ln \left(1+\frac{\Gamma_{T} \beta_{j+1, j}}{\beta_{j-1, j}}\right)+\ln \left(1+\frac{\Gamma_{T} \beta_{j, j}}{\beta_{j-1, j}}\right)\right)
\end{aligned}
$$

where $\beta_{i, j}=\frac{\lambda_{i, j} P_{i}}{N_{0}}$ with $\beta_{N+1, N}=0$, and $\beta_{N+2, N+1}=0$. Since we assume that directional antennas are used by the FD relays to reduce the loop interference and other degrading effects, we consider that $\lambda_{j+1, j}<\lambda_{j-1, j}$ (i.e., we assume that mean power gain of the IRI link at $F_{j}\left(\lambda_{j+1, j}\right)$ is less than the mean power gain of the desired link at $F_{j}\left(\lambda_{j-1, j}\right)$ ). Similarly, the mean power gain of the RSI link is assumed to be less than the mean channel gain of the desired link at $F_{j}$, i.e., $\lambda_{j, j}<\lambda_{j-1, j}$. The objective function can therefore be simplified using the approximation, $\ln (1+x) \approx$ $x, x<<1$, and the optimization problem can be re-casted as follows:

$$
\begin{aligned}
\underset{\substack{P_{j} \\
j=0,1, \ldots N}}{\operatorname{Min}} & F(\mathbf{P})=\sum_{j=1}^{N+1}\left(\frac{\Gamma_{T}}{\beta_{j-1, j}}+\frac{\Gamma_{T} \beta_{j+1, j}}{\beta_{j-1, j}}+\frac{\Gamma_{T} \beta_{j, j}}{\beta_{j-1, j}}\right) \\
\text { s.t. } & \sum_{j=0}^{N} P_{j} \leq P \\
& \sum_{j=0}^{N} P_{j} \lambda_{j, P R} \leq I_{\text {avg }} \\
& P_{j}>0, j=0,1,2, \ldots N
\end{aligned}
$$

Now, (31) is in the standard form of a geometric programming problem (GPP) [38]; a GPP in the standard form is represented as follows:

$$
\begin{aligned}
\operatorname{Min} & f_{0}(x) \\
\text { s.t. } & f_{i}(x) \leq 1, i=1,2 \ldots m \\
& h_{i}(x)=1, i=1,2 \ldots p
\end{aligned}
$$

where $f_{0}, f_{1}, \ldots f_{m}$ are posynomials and $h_{1}, h_{2} \ldots h_{p}$ are monomials. From (31), notice that the objective function is in posynomial form. The inequality constraints also have a posynomial form in the left hand side and a monomial form on the right hand side. Hence, (31) is a standard GPP which can be solved using standard convex optimization software tools [39]. Notice that average channel gains (i.e., statistical channel state information-SCSI) alone is needed for finding the OPA for problem (31). The numerical and simulation results for the outage probability are presented in Section 7.

\subsection{Instantaneous rate maximization in multi-hop CogFDR network}

Here, we investigate OPA to maximize the end-to-end instantaneous rate under a sum power constraint. Under the assumption that the inputs are complex Gaussian of unit bandwidth, with the secondary nodes employing a continuous rate scheme, we express the instantaneous transmission rate of the link between $F_{j-1}$ and $F_{j}$ as [40],

$$
R_{j}(\mathbf{P})=\log _{2}\left(1+\Gamma_{j, \mathrm{FDR}}^{(0)}(\mathbf{P})\right)
$$

where $\Gamma_{j, \mathrm{FDR}}^{(0)}(\mathbf{P}), \mathbf{P}=\left[P_{j}\right], j=0,1, \ldots N$, is given by (2) with $P_{N+1}=P_{N+2}=0$. The end-to-end instantaneous rate of the DF-based secondary network is expressed by the minimum of instantaneous rates of the $N+1$ links as [40],

$$
R(\mathbf{P})=\underset{j}{\operatorname{Min}} R_{j}(\mathbf{P}), \quad j=1,2, \ldots N+1
$$

Since the instantaneous rate is a function of instantaneous channel gains, the knowledge of instantaneous channel state information (ICSI) is required for solving the rate maximization problem. Along with this, we consider a peak interference constraint for the PR. Accordingly, the rate maximization problem is defined as follows:

$$
\begin{array}{ll}
\underset{\substack{P_{j} \\
j=0,1 . \ldots N}}{\operatorname{Max}} \operatorname{Min} & R_{j}(\mathbf{P})=\log _{2}\left(1+\Gamma_{j, \mathrm{FDR}}^{(0)}(\mathbf{P})\right) \\
\text { subject to } & \sum_{j=0}^{N} P_{j} \leq P \\
& \sum_{j=0}^{N} P_{j}\left|h_{j, P R}\right|^{2} \leq I \text { th } \\
& P_{j}>0, j=0,1, \ldots N
\end{array}
$$

Here, (35c) represents the peak interference power threshold for PR.

Lemma 1 In DF relaying under a total power constraint, maximizing the minimum of rates corresponding to the $N+1$ links in the system is equivalent to a condition where the instantaneous received SINRs over all the links become equal, i.e., $\Gamma_{j, F D R}^{(0)}(P)=\Gamma, \forall j=1,2, \ldots N+1$.

Proof The maximum achievable rate $R^{\max }(P)$ in a multihop relay network strictly increases with increase in total transmission power $P$ of the network. Let us assume that $P_{j}^{*}, j=\{0,1,2, . . N\}$ is the optimal power allocation for achieving a maximum rate of $R^{\max }(P)$. In contradiction to this statement, consider an arbitrary hop $k$ connecting nodes $F_{k-1}$ and $F_{k}$ having an instantaneous rate over this link, i.e., $R_{k}$ satisfying $R_{k}>R^{\max }(P)$. Since $R_{k}$ is an increasing function of $P_{k-1}$; let us slightly decrease $P_{k-1}$ such that $R_{k} \geq R^{\max }(P)$ is still unaffected. As $P_{k-1}$ decreases, the IRI from node $F_{k-1}$ to $F_{k-2}$ decreases 
which improves the received SINR at $F_{k-2}$, which in turn increases the rate $R_{k-2}$. To compensate for the increase in $R_{k-2}$, we can slightly reduce $P_{k-3}$, which in turn increases $R_{k-4}$ and so on. Therefore, with this new power allocation (i.e., with total power less than $P$ ), $R_{k} \geq R^{\max }(P)$ still holds. This contradicts the original property that $R^{\max }(P)$ is a strictly increasing function of $P$. This proves that the optimal rate over any given hop $F_{j}$ cannot be greater than $R^{\max }$, so the optimum power allocation corresponds to the condition for which the hop rates are equal, i.e., $R_{k}=R^{\max }(P)$. Since rate is given by the expression, $R_{j}(\mathbf{P})=\log _{2}\left(1+\Gamma_{j, \mathrm{FDR}}^{(0)}(\mathbf{P})\right)$, where $\log _{2}(x)$ is a monotonically increasing function of $x$, we can say that optimal power allocation which maximizes the minimum of rates is achieved by equating the rates, or equivalently, by equating the SINRs of all the links in the network.

To solve (35), we adopt the approach used in [40-42]. Therefore, the criteria for finding OPA which maximizes the end-to-end instantaneous rate of the secondary network, is given by,

$$
\Gamma_{j, \mathrm{FDR}}^{(0)}=\Gamma \quad \forall j=1,2, \ldots N+1
$$

Recall that the SINR at $F_{N+1}$ is given by (3b). Notice that $F_{N+1}$ does not suffer from RSI and IRI as is evident from (3b). The relay node $F_{N}$ does not suffer from IRI since its successor node $F_{N+1}$ being the destination does not transmit. However, $F_{N}$ suffers from RSI and hence the SINR at $F_{N}$ is given by (3a). The SINR at the remaining relay nodes $F_{j}, j=0,1, \ldots N-1$ are given by (2) in Section 3 of this paper. Combining (2) and (3) with (36), we get the following set of equations for the optimal transmit power $P_{j, \mathrm{FDR}}^{*}$ in terms of $\Gamma$ (i.e., the SINR per hop indicated in (36)):

$$
\begin{aligned}
P_{N, \mathrm{FDR}}^{*} & =\frac{\Gamma N_{0}}{\left|h_{N, N+1}\right|^{2}} \\
P_{N-1, \mathrm{FDR}}^{*} & =\frac{\Gamma N_{0}}{\left|h_{N-1, N}\right|^{2}}\left(1+\frac{\Gamma\left|h_{N, N}\right|^{2}}{\left|h_{N, N+1}\right|^{2}}\right) \\
P_{j-1, \mathrm{FDR}}^{*} & =\frac{\Gamma\left[N_{0}+P_{j, F D R}^{*}\left|h_{j j}\right|^{2}+P_{j+1, F D R}^{*}\left|h_{j+1, j}\right|^{2}\right]}{\left|h_{j-1, j}\right|^{2}} \\
j & =1,2, \ldots N
\end{aligned}
$$

where $P_{N+1, \mathrm{FDR}}^{*}=0$. Thus, we can see that the optimal value of the transmission power at $F_{j}, P_{j, \text { FDR }}^{*}$ can be recursively expressed as a function of $\Gamma$, i.e., $P_{j, \mathrm{FDR}}^{*}=g_{j}(\Gamma)$. Since the objective function given by (35a) is maximized when the constraints hold with equality [40], we can write the following equations for the constraints:

$$
\begin{aligned}
\sum_{j=0}^{N} g_{j}(\Gamma)\left|h_{j, P R}\right|^{2} & =I \text { th } \\
\sum_{j=0}^{N} g_{j}(\Gamma) & =P
\end{aligned}
$$

Notice that (38a) and (38b) are polynomial equations of order $N+1$, which needs to be solved to get $\Gamma$. Even though it is tough to determine the closed form solutions for $\Gamma$ from the above equations, we can use efficient numerical algorithms to find the solution [43]. Let $\Gamma_{1}$ and $\Gamma_{2}$ respectively be the solution for $\Gamma$ obtained from (38a) and (38b). Now, $\Gamma$ (i.e., SINR corresponding to the optimal powers) is chosen as $\Gamma=\min \left(\Gamma_{1}, \Gamma_{2}\right)$. Once $\Gamma$ is known, the optimal power values $P_{j, \mathrm{FDR}}^{*}, j=0,1, \ldots N$ can be determined as follows: First of all, we find $P_{N, F D R}^{*}$ using (37a). After that, we find $P_{j, \text { FDR }}^{*}, j=0,1, \ldots N-1$ using the recursive relations given by (37c). Further, notice that the complexity involved in the calculation of optimal transmit power is equivalent to that of finding the real roots of two polynomial equations of order $N+1$. Algorithm 1 illustrates the steps involved in determining the optimal transmit powers.

\subsubsection{Three-hop CogFDR network : a case study}

The end-to-end rate optimization problem for the threehop CogFDR network is expressed as follows:

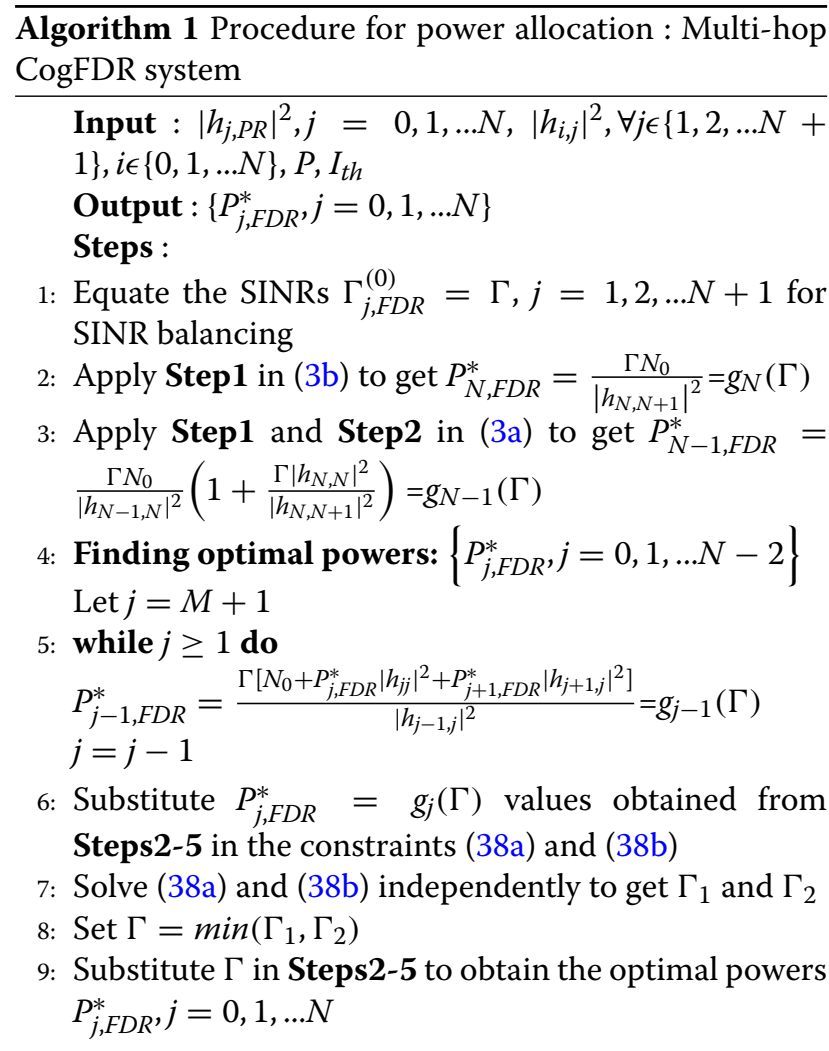




$$
\begin{array}{cl}
\underset{\substack{P_{j} \\
j=0,1,2}}{\operatorname{Max}} \operatorname{Min}_{j} & R_{j}(\mathbf{P})=\log _{2}\left(1+\Gamma_{j, \mathrm{FDR}}^{(0)}(\mathbf{P})\right) \\
\text { s.t. } & P_{0}\left|h_{0, P R}\right|^{2}+P_{1}\left|h_{1, P R}\right|^{2}+P_{2}\left|h_{2, P R}\right|^{2} \leq I \text { th } \\
& \sum_{j=0}^{2} P_{j} \leq P \\
& P_{j}>0, j=0,1,2
\end{array}
$$

Let $\Gamma_{j, \mathrm{FDR}}^{(0)}, j=1,2,3$ be the SINR/SNR at the relay nodes $F_{1}, F_{2}$, and $F_{3}$, respectively. Using the fact that the endto-end rate is maximized when the hop SINRs are equal (under total power constraint), we have,

$$
\Gamma_{1, F D R}^{(0)}=\Gamma_{2, F D R}^{(0)}=\Gamma_{3, F D R}^{(0)}=\Gamma
$$

where

$$
\begin{aligned}
\Gamma_{1, \mathrm{FDR}}^{(0)} & =\frac{P_{0}\left|h_{01}\right|^{2}}{N_{0}+P_{1}\left|h_{11}\right|^{2}+P_{2}\left|h_{21}\right|^{2}} \\
\Gamma_{2, \mathrm{FDR}}^{(0)} & =\frac{P_{1}\left|h_{12}\right|^{2}}{N_{0}+P_{2}\left|h_{22}\right|^{2}} \\
\Gamma_{3, \mathrm{FDR}}^{(0)} & =\frac{P_{2}\left|h_{23}\right|^{2}}{N_{0}}
\end{aligned}
$$

From (41), we get the optimal power values as:

$$
\begin{aligned}
& P_{2, \mathrm{FDR}}^{*}=\frac{\Gamma N_{0}}{\left|h_{2,3}\right|^{2}} \\
& P_{1, \mathrm{FDR}}^{*}=\frac{\Gamma N_{0}}{\left|h_{1,2}\right|^{2}}\left(1+\frac{\Gamma\left|h_{2,2}\right|^{2}}{\left|h_{2,3}\right|^{2}}\right) \\
& P_{0, \mathrm{FDR}}^{*}=\frac{\Gamma N_{0}}{\left|h_{0,1}\right|^{2}}\left[1+\frac{\Gamma\left|h_{1,1}\right|^{2}}{\left|h_{1,2}\right|^{2}}\left(1+\frac{\Gamma\left|h_{2,2}\right|^{2}}{\left|h_{2,3}\right|^{2}}\right)+\frac{\Gamma\left|h_{2,1}\right|^{2}}{\left|h_{2,3}\right|^{2}}\right]
\end{aligned}
$$

Since $P_{j, \mathrm{FDR}}^{*}=g_{j}(\Gamma), j=0,1,2$, we write the constraints of (39) as:

$$
\begin{aligned}
\sum_{j=0}^{2} g_{j}(\Gamma)\left|h_{j, P R}\right|^{2} & =I \text { th } \\
\sum_{j=0}^{2} g_{j}(\Gamma) & =P
\end{aligned}
$$

Substituting $P_{j, \mathrm{FDR}}^{*},\{i=0,1,2\}$ given by (42) in (43), the following pair of cubic equations are obtained:

$$
\begin{aligned}
& \Gamma^{3} N_{0}\left(\frac{\left|h_{0, P R}\right|^{2}}{\left|h_{0,1}\right|^{2}} \frac{\left|h_{1,1}\right|^{2}}{\left|h_{1,2}\right|^{2}} \frac{\left|h_{2,2}\right|^{2}}{\left|h_{2,3}\right|^{2}}\right) \\
& +\Gamma^{2} N_{0}\left(\frac{\left|h_{0, P R}\right|^{2}}{\left|h_{0,1}\right|^{2}}\left(\frac{\left|h_{1,1}\right|^{2}}{\left|h_{1,2}\right|^{2}}+\frac{\left|h_{2,1}\right|^{2}}{\left|h_{2,3}\right|^{2}}\right)+\frac{\left|h_{1, P R}\right|^{2}}{\left|h_{1,2}\right|^{2}} \frac{\left|h_{2,2}\right|^{2}}{\left|h_{2,3}\right|^{2}}\right) \\
& +\Gamma N_{0}\left(\frac{\left|h_{0, P R}\right|^{2}}{\left|h_{0,1}\right|^{2}}+\frac{\left|h_{1, P R}\right|^{2}}{\left|h_{1,2}\right|^{2}}+\frac{\left|h_{2, P R}\right|^{2}}{\left|h_{2,3}\right|^{2}}\right)=I \text { th } \quad(44 \mathrm{a}) \\
& \Gamma^{3} N_{0}\left(\frac{\left|h_{1,1}\right|^{2}}{\left|h_{0,1}\right|^{2}\left|h_{1,2}\right|^{2}} \frac{\left|h_{2,2}\right|^{2}}{\left|h_{2,3}\right|^{2}}\right) \\
& +\Gamma^{2} N_{0}\left(\frac{\left|h_{1,1}\right|^{2}}{\left|h_{0,1}\right|^{2}\left|h_{1,2}\right|^{2}}+\frac{\left|h_{2,1}\right|^{2}}{\left|h_{0,1}\right|^{2}\left|h_{2,3}\right|^{2}}+\frac{\left|h_{2,2}\right|^{2}}{\left|h_{1,2}\right|^{2}\left|h_{2,3}\right|^{2}}\right) \\
& +\Gamma N_{0}\left(\frac{1}{\left|h_{0,1}\right|^{2}}+\frac{1}{\left|h_{1,2}\right|^{2}}+\frac{1}{\left|h_{2,3}\right|^{2}}\right)=P
\end{aligned}
$$

Let $\Gamma_{1}$ and $\Gamma_{2}$ be the solution corresponding to (44a) and (44b), respectively. Then $\Gamma$ is chosen as $\Gamma=\min \left(\Gamma_{1}, \Gamma_{2}\right)$. Substituting $\Gamma$ in $(42 a)-(42 c)$, we can find the optimal powers $P_{i, \mathrm{FDR}}^{*},\{i=0,1,2\}$.

\section{Optimal power allocation (OPA) for the secondary network with primary interference}

In this section, we determine the OPA that either minimizes the end-to-end outage probability or maximizes the end-to-end instantaneous rate of the secondary network under the influence of interference from PT.

\subsection{Outage probability minimization in multi-hop CogFDR network with primary interference}

Here, we follow the approach adopted in Section 5.1. To minimize $P_{\text {out,FDR }}^{(1)}$ derived in Section 4.1.2, we consider the function $F_{1}(\mathbf{P})=-\ln \left(1-P_{\text {out,FDR }}^{(1)}\right)$. Utilizing (21) and (23a) $F_{1}(\mathbf{P})$ can be written as,

$$
\begin{aligned}
F_{1}(\mathbf{P}) & =-\ln \left[1-\left(1-\prod_{j=1}^{N+1} \operatorname{Pr}\left(\Gamma_{j, \mathrm{FDR}}^{(1)} \geq \Gamma_{T}\right)\right)\right] \\
& =-\ln \left[\prod_{j=1}^{N+1} \operatorname{Pr}\left(\Gamma_{j, \mathrm{FDR}}^{(1)} \geq \Gamma_{T}\right)\right] \\
& =-\sum_{j=1}^{N+1}\left[-\frac{\Gamma_{T}}{\beta_{j-1, j}}-m \ln \left(\frac{\Gamma_{T} \beta}{\beta_{j-1, j}}+1\right)\right]
\end{aligned}
$$

where $m$ and $\beta$ are defined in (23b) and (23c), respectively. The objective function $F_{1}(\mathbf{P})$ is not in the standard posynomial form of a GPP. We can convert the function in (45) in the standard form by using the approximation $\ln (1+x) \approx x$ when $x$ is small. Here, assume that the mean channel gain of the desired signal $\lambda_{j-1, j}$ is greater than the interference link channel gains $\lambda_{j, j}, \lambda_{j+1, j}$ and 
$\lambda_{P T, j}$. Intuitively, $\beta_{j-1, j}>\beta$, and thus, we can approximate $\ln \left(1+\Gamma_{T} \beta / \beta_{j-1, j}\right) \approx \Gamma_{T} \beta / \beta_{j-1, j}$. Therefore, we can further simplify $F_{1}(\mathbf{P})$ as follows:

$$
\begin{aligned}
F_{1}(\mathbf{P}) & =\sum_{j=1}^{N+1}\left[\frac{\Gamma_{T}}{\beta_{j-1, j}}+m \frac{\Gamma_{T} \beta}{\beta_{j-1, j}}\right] \\
& =\sum_{j=1}^{N+1}\left[\frac{\Gamma_{T}}{\beta_{j-1, j}}+\frac{\Gamma_{T}}{\beta_{j-1, j}}\left(\beta_{j, j}+\beta_{j+1, j}+\beta_{P T, j}\right)\right] \\
& =\sum_{j=1}^{N+1} \frac{\Gamma_{T}}{\beta_{j-1, j}}\left(1+\beta_{j, j}+\beta_{j+1, j}+\beta_{P T, j}\right)
\end{aligned}
$$

Now, the objective function $F_{1}(\mathbf{P})$ is in the standard posynomial form. The optimization problem under the influence of interference from PT is given by,

$$
\begin{aligned}
\operatorname{Min}_{\substack{P_{j} \\
j=0,1, \ldots N}} & F_{1}(\mathbf{P})=\sum_{j=1}^{N+1} \frac{\Gamma_{T}}{\beta_{j-1, j}}\left(1+\beta_{j, j}+\beta_{j+1, j}+\beta_{P T, j}\right) \\
\text { s.t. } & \sum_{j=0}^{N} P_{j} \leq P \\
& \sum_{j=0}^{N} P_{j} \lambda_{j, P R} \leq I_{\mathrm{avg}} \\
& P_{j}>0, j=0,1,2, \ldots N
\end{aligned}
$$

Now, (47) is a standard GPP, which can be solved using Convex optimization software tools [39].

\subsection{Rate maximization in multi-hop CogFDR network with primary interference}

By following the approach detailed in Section 5.2, the OPA problem for instantaneous rate maximization is formulated as follows:

$$
\begin{aligned}
\underset{\substack{P_{j} \\
j=0,1, \ldots N}}{\operatorname{Max}} \operatorname{Min}_{j} & R_{j}(\mathbf{P})=\log _{2}\left(1+\Gamma_{j, \mathrm{FDR}}^{(1)}(\mathbf{P})\right) \\
\text { s.t. } & \sum_{j=0}^{N} P_{j} \leq P \\
& \sum_{j=0}^{N} P_{j}\left|h_{i, P R}\right|^{2} \leq I \text { th } \\
& P_{j}>0, j=0,1, \ldots N
\end{aligned}
$$

As detailed in Section 5.2, for solving (48), we adopt the approach in [40-42], where the criterion for instantaneous rate maximization is that the instantaneous SINRs over each hop must be made equal.

$$
\Gamma_{j, \mathrm{FDR}}^{(1)}=\Gamma_{0}, j=1,2, \ldots N+1
$$

where $\Gamma_{j, \mathrm{FDR}}^{(1)}, j=1,2, \ldots N+1$ is given by (19) with $P_{N+1}$ $=P_{N+2}=0$. Applying (49), the optimal powers can be determined as follows:

$$
\begin{aligned}
& P_{N, \mathrm{FDR}}^{*}=\frac{\Gamma_{0}}{\left|h_{N, N+1}\right|^{2}}\left(N_{0}+P_{P T}\left|h_{P T, N+1}\right|^{2}\right) \\
& P_{N-1, \mathrm{FDR}}^{*}=\frac{\Gamma_{0}}{\left|h_{N-1, N}\right|^{2}}\left(N_{0}+\frac{\Gamma_{0}\left|h_{N, N}\right|^{2}}{\left|h_{N, N+1}\right|^{2}}\left(N_{0}+P_{P T}\left|h_{P T, N+1}\right|^{2}\right)+P_{P T}\left|h_{P T, N}\right|^{2}\right) \\
& P_{j-1, \mathrm{FDR}}^{*}= \frac{\Gamma_{0}}{\left|h_{j-1, j}\right|^{2}}\left(N_{0}+P_{j, \mathrm{FDR}}^{*}\left|h_{j, j}\right|^{2}+P_{j+1, \mathrm{FDR}}^{*}\left|h_{j+1, j}\right|^{2}+P_{P T}\left|h_{P T, j}\right|^{2}\right), \\
& j=1,2, \ldots N, P_{N+1}=0
\end{aligned}
$$

Notice that $P_{j, \mathrm{FDR}}^{*}$ is a function of $\Gamma_{0}$, i.e., $P_{j, \mathrm{FDR}}^{*}=q_{j}\left(\Gamma_{0}\right)$. Thus, the constraints (48b) and (48c) can be written as follows:

$$
\begin{aligned}
\sum_{j=0}^{N} q_{j}\left(\Gamma_{0}\right)\left|h_{j, P R}\right|^{2} & =I \text { th } \\
\sum_{j=0}^{N} q_{j}\left(\Gamma_{0}\right) & =P
\end{aligned}
$$

The polynomial Eqs. (51a) and (51b) can be solved to find $\Gamma_{0}$. Let $\Gamma_{01}$ and $\Gamma_{02}$ be the solution for $\Gamma_{0}$ obtained from (51a) and (51b), respectively. Then, $\Gamma_{0}=\min \left(\Gamma_{01}, \Gamma_{02}\right)$. The knowledge of $\Gamma_{0}$ and ICSI will enable us to find $P_{j, \mathrm{FDR}}^{*}$ using (50).

\section{Results and discussion}

Here, we present the theoretical and simulation results for the outage and rate performance of multi-hop CogFDR/HDR networks considering the proposed OPA and the conventional EPA strategies. We consider a threehop secondary network where the secondary nodes $F_{j}$, $j=0,1,2,3$ are assumed to be located on a straight line at $(-1.5,0),(-0.5,0),(0.5,0)$, and $(1.5,0)$, respectively. The positions of PT and PR are assumed to be at $(-1.5,1)$ and $(-0.5,1)$, respectively. The RSI at the secondary relays are assumed as equal to $-40 \mathrm{~dB}$ (unless otherwise specified). We select the path loss exponent $(n)$ to be equal to four for all the links in the network. The channel gains are defined as $\lambda_{i, j}=G D_{i, j}^{-n}$; here, $G=1$ and the distances $D_{i, j}$ are calculated from the respective location coordinates. The target rate is chosen as $r=0.1 \mathrm{bits} / \mathrm{sec} / \mathrm{Hz}$. The distance between $\mathrm{ST}$ and SR is fixed as equal to $3 \mathrm{~km}$ regardless of the number of relays in the network. The analytical findings are verified by extensive Monte Carlo simulations. Each point in the simulation result is obtained by averaging the results of $10^{5}$ simulation runs. In all figures, 
analytical plots are identified by lines and simulation plots by markers. We consider the EPA scheme as well, for which the transmit powers $P_{j}, j=1,2, \ldots N$ corresponding to the secondary nodes in CogFDR network are set as given by (13).

Figure 2 depicts the outage probability comparison of a three-hop CogFDR and CogHDR network under EPA against $P / N_{0}$ which is the ratio of total transmit power-tonoise power. Here, we consider distinct values for $I_{\text {avg }} / N_{0}$, the ratio of tolerable average interference-to-noise power ratio. In the low transmit power region, CogFDR performs better than CogHDR networks because the target data rate requirement for $\operatorname{CogHDR}$ is higher than that of CogFDR. However, as $P / N_{0}$ increases, CogHDR networks show improved performance as compared to CogFDR networks due to the fact that higher transmit power increases the RSI and IRI in the case of FDR networks. Both CogHDR and CogFDR networks suffer from an outage floor behavior at high transmit powers, as seen in Fig. 2. In the case of CogHDR and CogFDR networks, the outage floor occurs owing to the fact that the transmit power depends on the tolerable interference threshold at the PR and the total transmit power according to (13). As a result, for a given $I_{\text {avg, }}$, if we continue to increase the transmit power, proportional change in outage will not be observed. Apart from this, CogFDR networks suffer from degradation in outage performance at higher transmit powers due to the presence of RSI and IRI. Due to this, the outage floor in CogFDR networks occur for comparatively lower values of $P / N_{0}$ as can be seen in Fig. 2 . When the tolerable interference power $\left(I_{\text {avg }}\right)$ at the PR increases, it is observed that the outage decreases because the nodes can transmit at higher power; however, the rate of decrease is very small due to the proportional increase in RSI and IRI.

Figure 3 presents the impact of RSI on outage probability of $\operatorname{CogFDR}$ network under EPA. Here, we assume the mean RSI power at the secondary nodes $F_{0}, F_{1}$, and $F_{2}$ to be equal and denote it as $\lambda_{r r}$. When RSI decreases, the outage probability of CogFDR network decreases. With $P / N_{0}=20 \mathrm{~dB}$, the outage probability reduces by almost $74 \%$ when the RSI decreases from $-5 \mathrm{~dB}$ to $-35 \mathrm{~dB}$. In other words, the range of transmit power values over which the outage probability of CogFDR network is lower than that of CogHDR network, decreases as RSI becomes higher.

Figure 4 plots outage probability versus $I_{\text {avg }} / N_{0}$, for n values of $P / N_{0}$. When $P / N_{0}=15 \mathrm{~dB}, \operatorname{CogFDR}$ outperforms CogHDR for all values of $I_{\mathrm{avg}} / N_{0}$ considered. How ever, when $P / N_{0}$ is increased to $25 \mathrm{~dB}$, CogFDR outage probability is higher than that of CogHDR network due to the severe effects of RSI and IRI in the high transmit power region.

Figures 5, 6, 7, and 8 show the outage probability performance of CogFDR networks under OPA and EPA. In Fig. 5, the outage probability is drawn against $P / N_{0}$. From the graph, it is clear that OPA leads to improvement in the outage performance of CogFDR networks in

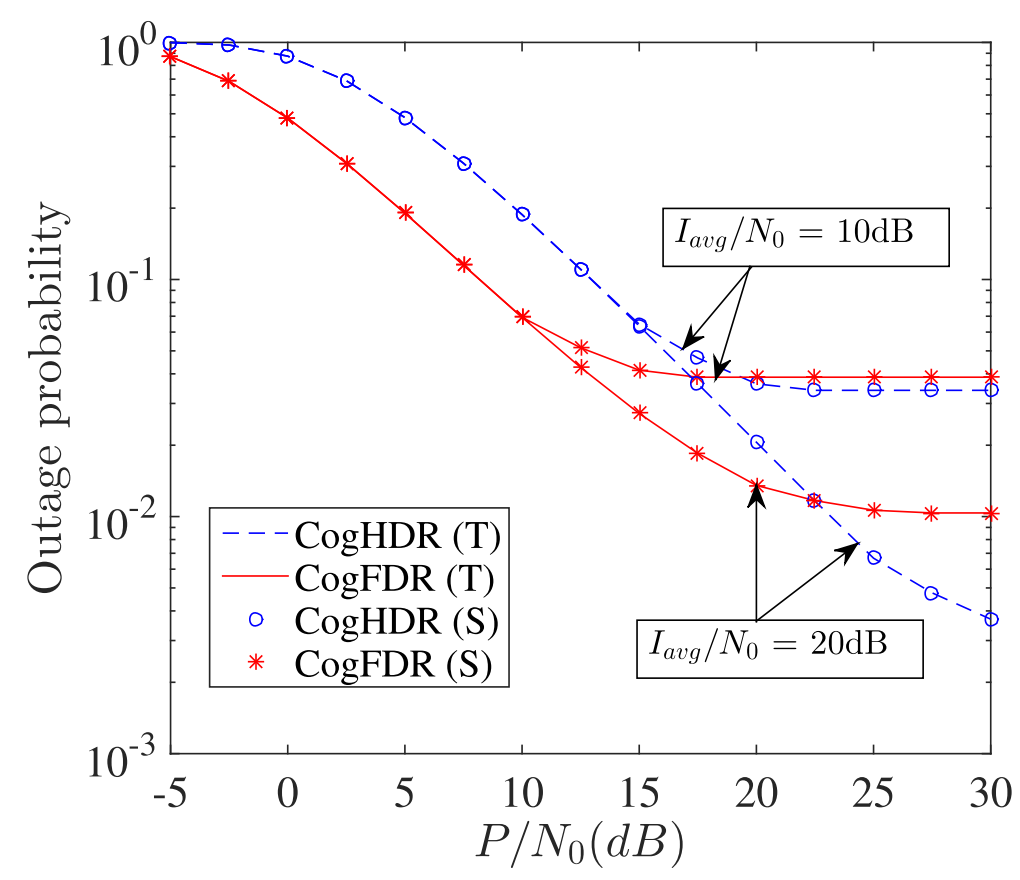

Fig. 2 Outage probability vs. $P / N_{0}$ for $l_{\text {avg }} / N_{0}=10 \mathrm{~dB}$ and $20 \mathrm{~dB}\left(\lambda_{r r}=-40 \mathrm{~dB}\right)$ 


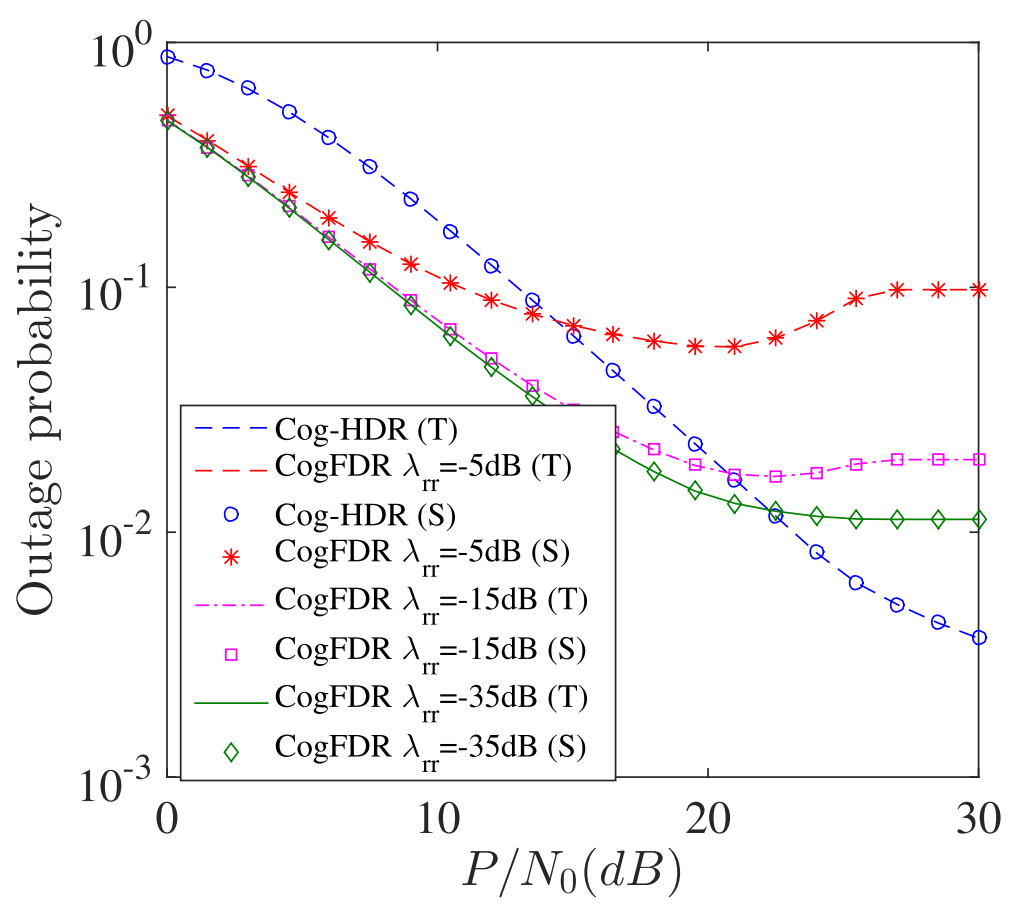

Fig. 3 Outage probability vs. $P / N_{0} ; l_{\text {avg }} / N_{0}=20 \mathrm{~dB}\left(\lambda_{r r}=-40 \mathrm{~dB}\right)$

comparison to that of EPA. In the high transmit power region, CogHDR network continues to show better performance than CogFDR-OPA. In Fig. 5, we can observe that the range over which CogFDR-OPA performs better than CogHDR-EPA is increased by $5 \mathrm{~dB}$, in the high transmit power region, as compared to CogFDR-EPA. With $P / N_{0}=25 \mathrm{~dB}$, OPA reduces the outage probability of CogFDR system by almost $50 \%$ as compared to EPA. For CogFDR, the OPA distributes the available transmit power among the secondary nodes of the CogFDR

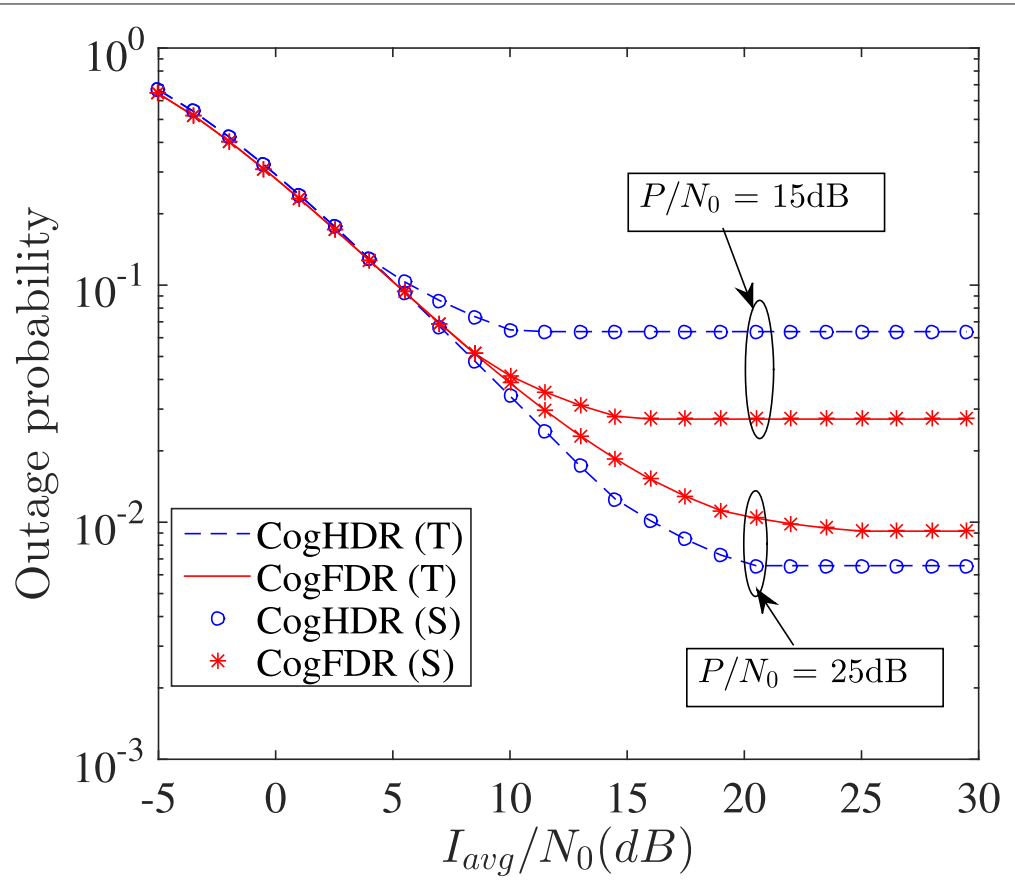

Fig. 4 Outage probability vs. lavg $/ N_{0}$ for $P / N_{0}=15 \mathrm{~dB}$ and $25 \mathrm{~dB}$ 


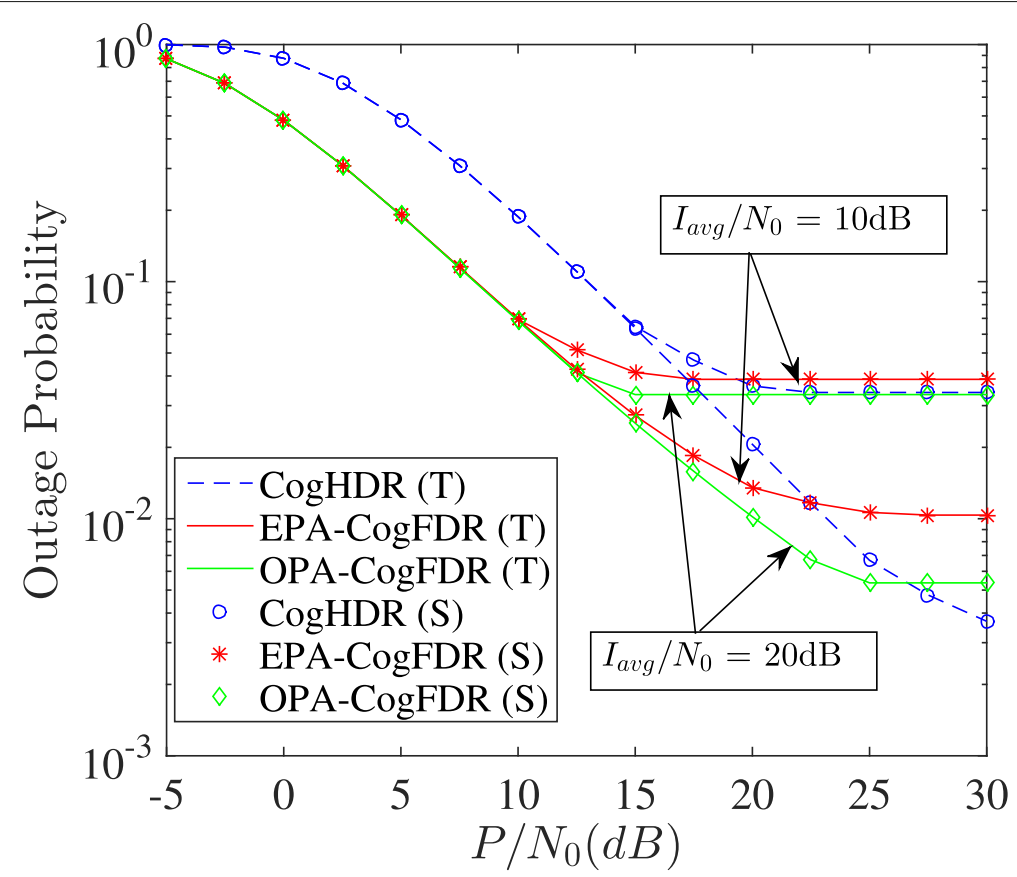

Fig. 5 Outage probability comparison with varying interference threshold power; $P / N_{0}=20 \mathrm{~dB}\left(\lambda_{r r}=-40 \mathrm{~dB}\right)$

network such that the effects of RSI and IRI are reduced. Even though the outage floor still exists in the high transmit power region, the benefit of OPA is that the value of $P / N_{0}$ at which the floor occurs is extended, as can be seen in Fig. 5. The results in Fig. 6 show that the range of $P / N_{0}$ values over which CogFDR performs better than CogHDR improves when the mean RSI power is reduced.

Figure 7 depicts the outage probability comparison plots with respect to target rate variations. With increasing target rate, the outage performance is badly affected,

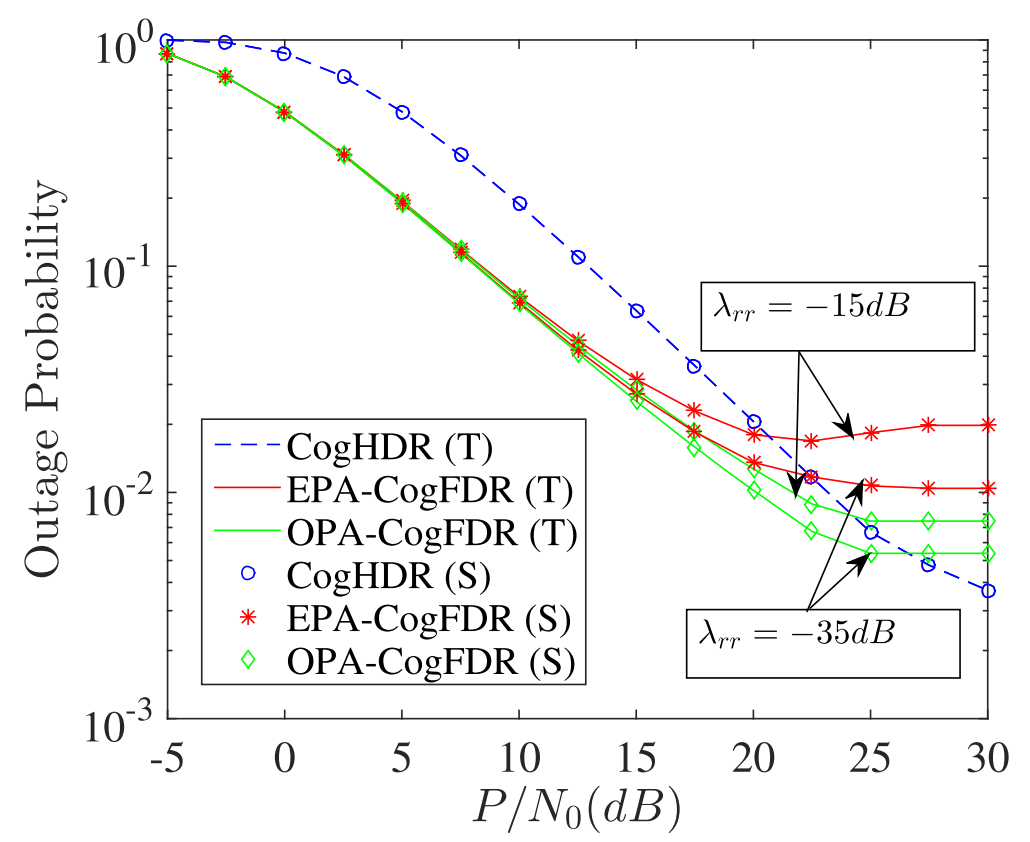

Fig. 6 Outage probability comparison with varying RSI; lavg $/ N_{0}=20 \mathrm{~dB}$ 


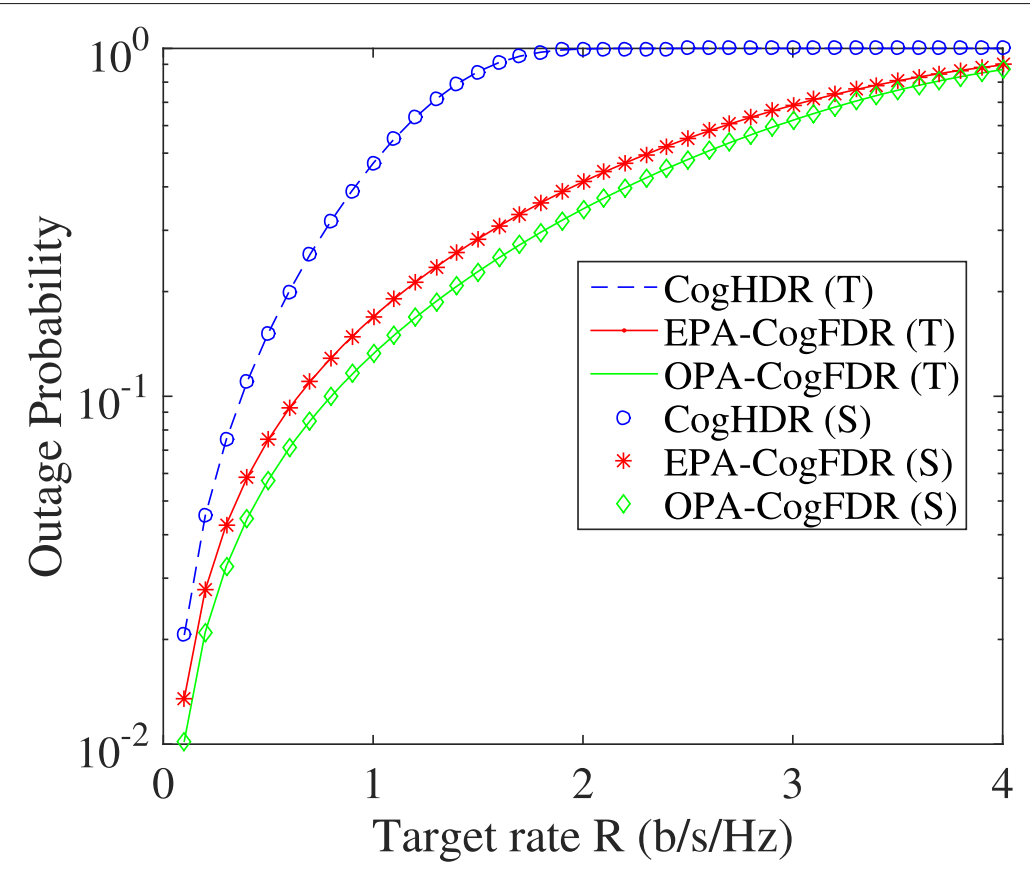

Fig. 7 Outage probability vs. $R ; P / N_{0}=20 \mathrm{~dB} ; l_{\text {avg }} / N_{0}=20 \mathrm{~dB}$

since the SINR threshold is also increased. However, OPA leads to performance improvement in outage. At $P / N_{0}=20 \mathrm{~dB}$, the performance improvement in outage of CogFDR system is $21.6 \%$ as compared to the equivalent EPA case.
Table 1 shows the OPA vector, i.e., $P_{j, \mathrm{FDR}}^{*} / N_{0}, j=0,1,2$, the normalized transmit powers, for two distinct values of $P / N_{0}$ under $\delta=-3 \mathrm{~dB},-5 \mathrm{~dB}$, and $-7 \mathrm{~dB}$, respectively. Here, $\delta$ represents the level of isolation provided by the directional antennas, for reducing the effect of IRI. Notice

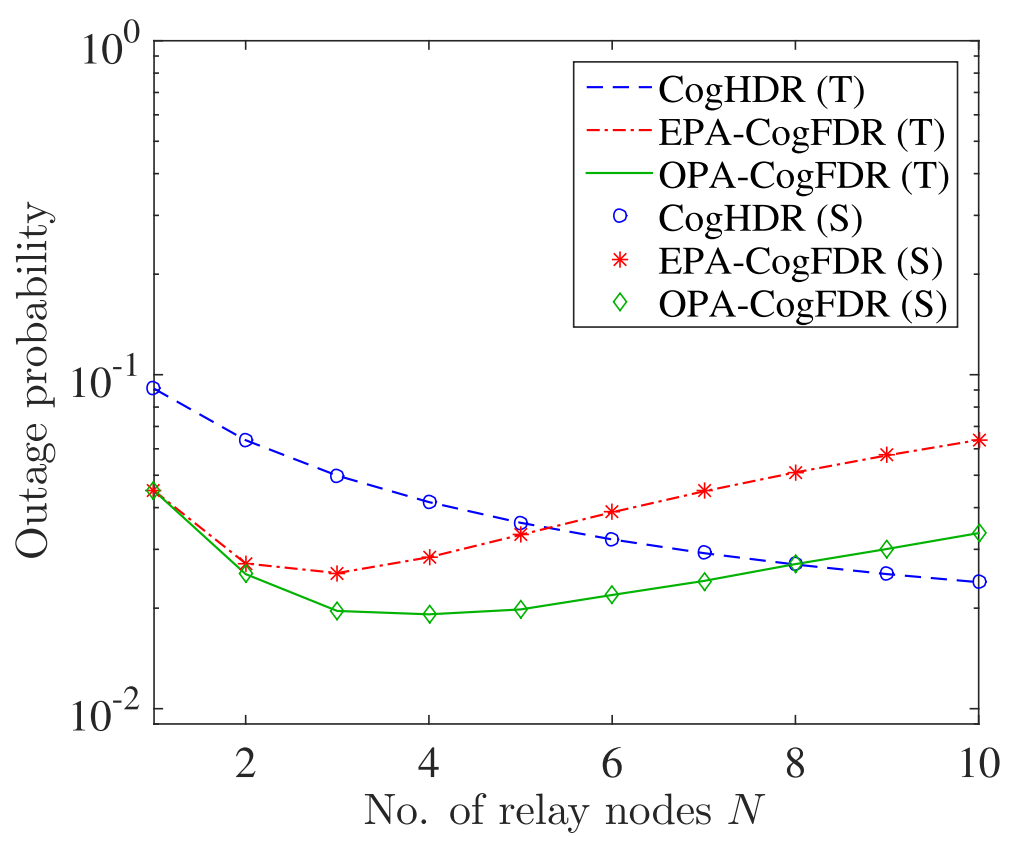

Fig. 8 Outage probability comparison for different number of relay nodes; $P / N_{0}=15 \mathrm{~dB}, I_{\text {avg }} / N_{0}=15 \mathrm{~dB}$ 
Table 1 Comparison of power allocation schemes for various levels of IRI $\delta$ and total transmit power $P / N_{0}$

\begin{tabular}{|c|c|c|c|c|}
\hline \multirow[t]{3}{*}{$\delta$} & \multicolumn{4}{|c|}{$P_{j, \mathrm{FDR}}^{*} / N_{0}, j=\{0,1,2\}$} \\
\hline & \multicolumn{2}{|l|}{$P / N_{0}=10 \mathrm{~dB}$} & \multicolumn{2}{|l|}{$P / N_{0}=20 \mathrm{~dB}$} \\
\hline & CogFDR-EPA & CogFDR-OPA & CogFDR-EPA & CogFDR-OPA \\
\hline$-3 \mathrm{~dB}$ & $3.33,3.33,3.33$ & $4.62,3.18,2.19$ & $33.33,33.33,33.33$ & $64.06,25.66,10.27$ \\
\hline$-5 \mathrm{~dB}$ & $3.33,3.33,3.33$ & $4.32,3.24,2.43$ & $33.33,33.33,33.33$ & $60.56,27.21,12.22$ \\
\hline$-7 \mathrm{~dB}$ & $3.33,3.33,3.33$ & $4.07,3.28,2.64$ & $33.33,33.33,33.33$ & $56.98,28.63,14.38$ \\
\hline
\end{tabular}

that for EPA scheme, the total available transmit power is divided equally among the nodes. The OPA for the CogFDR network is such that the power is allocated in the descending order for nodes $F_{0}, F_{1}$, and $F_{2}$. Since $F_{3}$ is the destination node, it does not transmit and does not have RSI either. So the transmit power for $F_{2}$ is comparatively lower. However, $F_{2}$ suffers from RSI and contributes to IRI for $F_{1}$, which is taken care by allocating more power to $F_{1}$. Due to this, the RSI at $F_{1}$ increases, the effect of which is compensated by allocating higher power to $F_{0}$. This is clearly depicted in Table 1 . As the IRI channel gain is reduced by $\delta=-7 \mathrm{~dB}$, the interference caused by $R_{2}$ on $R_{1}$ is reduced. Hence, $P_{2, \mathrm{FDR}}^{*} / N_{0}$ can be increased in order to improve the SINR of $R_{2}-R_{3}$ link causing a small interference effect at $R_{1}$. This small increase in interference at $R_{1}$ is compensated by slightly increasing the power $P_{1, \mathrm{FDR}}^{*} / N_{0}$ as well.

Figure 8 shows the outage probabilities of CogFDR network under EPA and OPA versus the number of relays. The RSI level at all the nodes has been assumed as equal to $-40 \mathrm{~dB}$. The separation between $F_{0}$ and $F_{N+1}$ is assumed to be constant. Accordingly, the number of hops significantly affects the outage performance of the secondary network. Initially for CogFDR, when $N$ increases, the outage decreases owing to the reduced link distance. However, when $N$ becomes larger, each node introduce additional RSI and IRI into the system so that the outage performance degradation happens. Notice that with increase in number of relays, the outage probability decreases monotonically in CogHDR due to reduced path

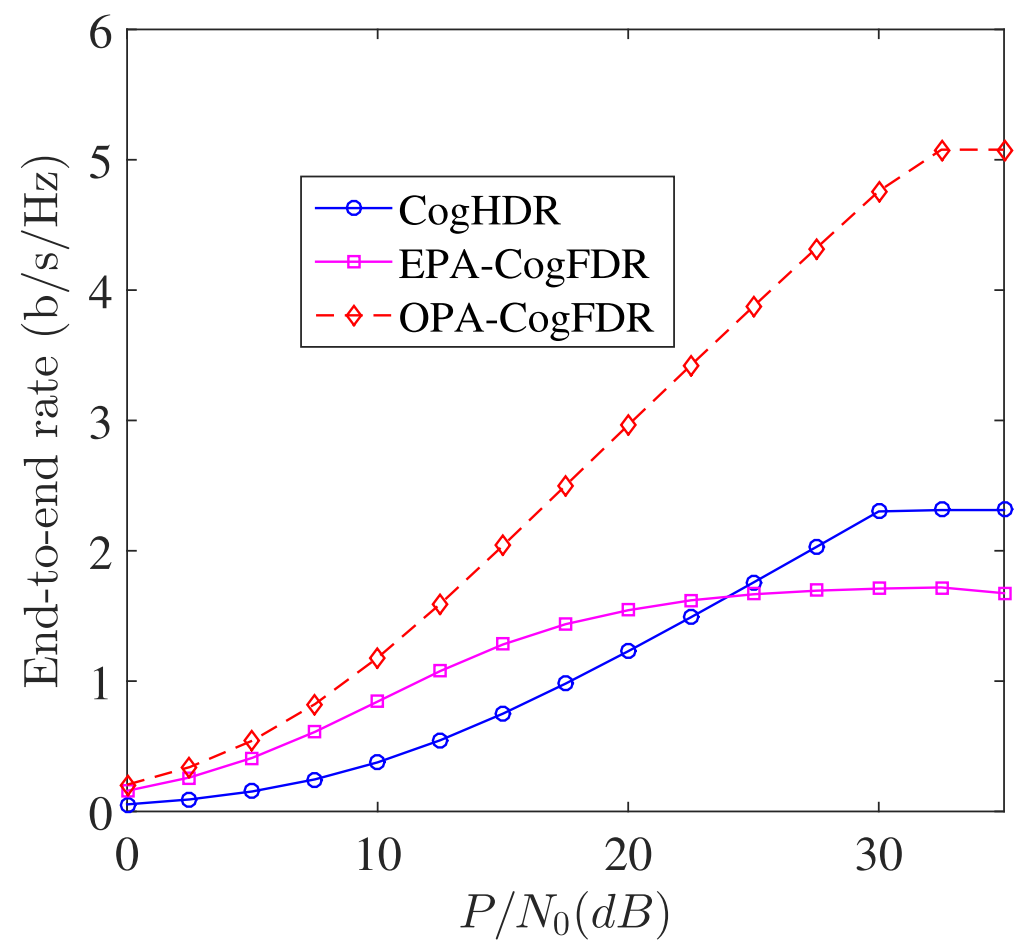

Fig. 9 Instantaneous end-to-end rate comparison; $\lambda_{r r}=-25 \mathrm{~dB}, I_{\text {th }} / N_{0}=20 \mathrm{~dB}$ 
loss between relay links. Further CogFDR-OPA outperforms CogFDR-EPA.

Figure 9 shows the maximum instantaneous end-to-end rate for the three-hop secondary network of CogFDR system under EPA/OPA schemes. Here, we choose the EPA powers for CogHDR and CogFDR as follows:

$$
\begin{aligned}
& P_{j, \mathrm{HDR}}=\min \left(\frac{P}{N+1}, \frac{I \mathrm{th}}{\left|h_{j, P R}\right|^{2}}\right) \\
& P_{j, \mathrm{FDR}}=\min \left(\frac{P}{N+1}, \frac{I \text { th }}{(N+1)\left|h_{j, P R}\right|^{2}}\right)
\end{aligned}
$$

The results show that OPA improves the instantaneous end-to-end rate of FDR system. When $P / N_{0}=30 \mathrm{~dB}$, the improvement in instantaneous rate is found to be $135 \%$ as compared to EPA. Further, it can be seen that, when $P / N_{0}$ is lower, instantaneous rate increases; however, for larger $P / N_{0}$, the rate is found to be almost saturating. For CogHDR and CogFDR-EPA, this saturation behavior is due to the transmit power limitation imposed by the condition given in (52) and (53) respectively. As the normalized transmit power $P / N_{0}$ becomes very large, the instantaneous transmit power becomes a function of $I$ th, which is fixed as a constant. Hence, the rate is no longer a function of $P$, and therefore, saturation occurs. For CogFDR-EPA system, saturation happens for lower $P / N_{0}$ as compared to CogHDR-EPA, due to the presence of RSI and IRI in the FDR system. In the case of CogFDROPA, the optimal distribution of transmit power among the nodes leads to significant improvement in the date rate. To determine the instantaneous rate corresponding to OPA/EPA, the following channel coefficient matrix has been assumed.

$$
H=\left[\begin{array}{lll}
0.3661 & 0 & 0 \\
0.0032 & 1.2156 & 0 \\
0.1579 & 0.0037 & 0.3567
\end{array}\right]
$$

In this case, each element $\left|h_{i, j}\right|^{2}, i=\{0,1,2\}, j=\{1,2,3\}$ is obtained by generating exponential random variables of a given mean value, which depends on the distance $D_{i, j}$ and path loss exponent $n$ (assumed to be equal to 4). Further, the channel coefficients for the secondary network to PR links have been assumed as,

$$
H_{P R}=\left[\begin{array}{lll}
0.0430 & 1 & 0.0410
\end{array}\right]
$$

where the elements denote $\left|h_{j, P R}\right|^{2}, j=\{0,1,2\}$, which are also generated from exponential random variables. The OPA vector and the corresponding instantaneous rate have been determined by following the method described in Section 5.2.

Figure 10 presents the plot for end-to-end instantaneous rate versus $I_{\text {th }} / N_{0}$ when $P / N_{0}=25 \mathrm{~dB}$. It can be seen from the graph that OPA improves the instantaneous rate of CogFDR network as compared to EPA. Further, we can observe that saturation behavior happens when $I_{\text {th }} / N_{0}$ is

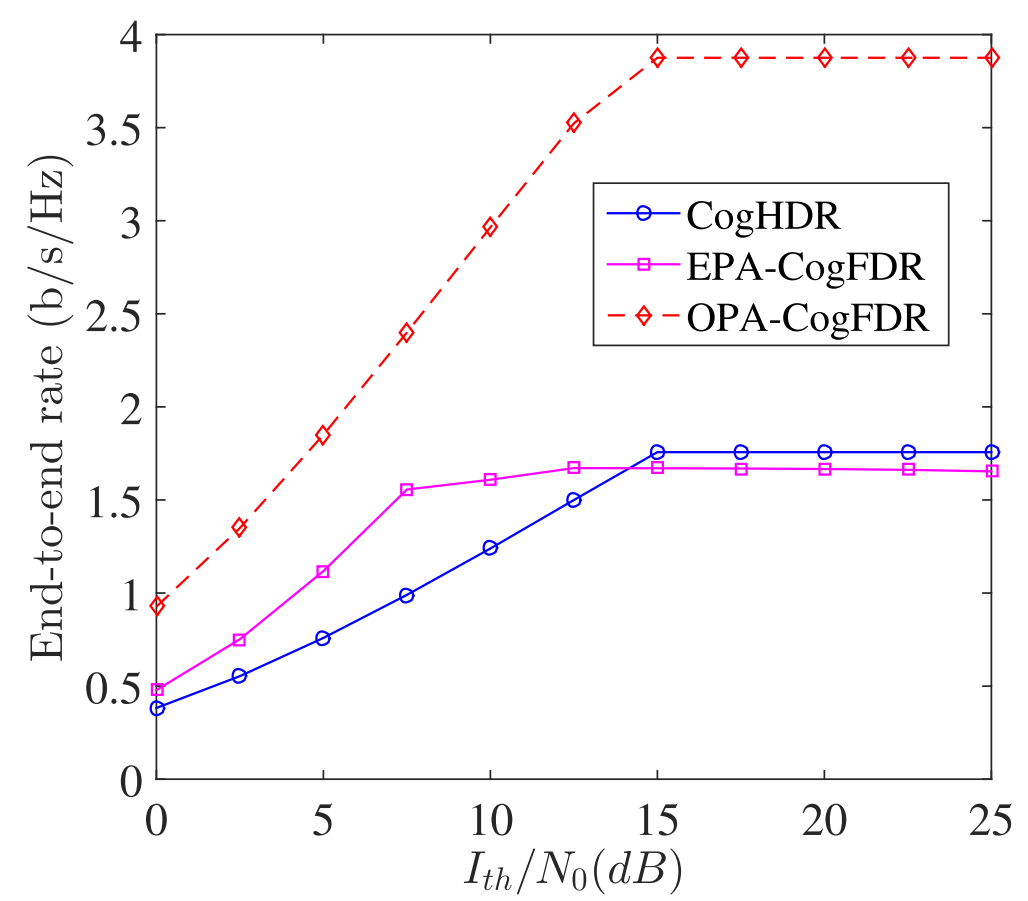

Fig. 10 End-to-end instantaneous rate comparison w.r.t. /th $/ N_{0} ; P / N_{0}=25 \mathrm{~dB}$ 


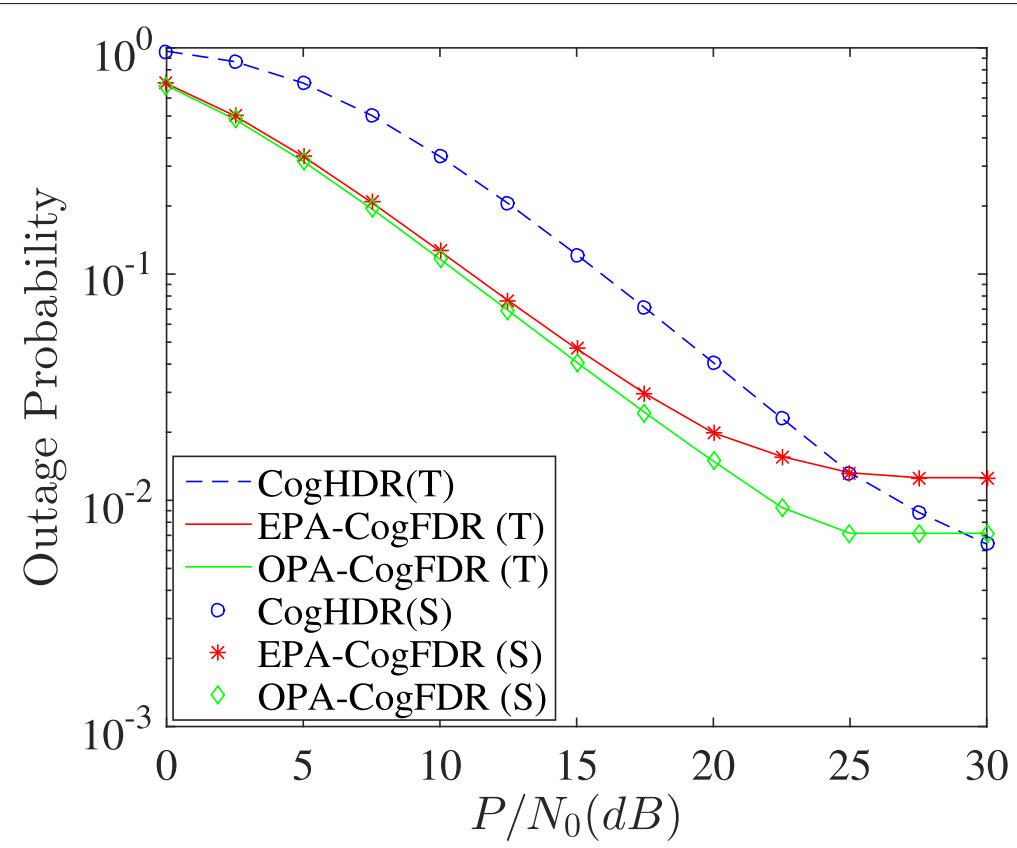

Fig. 11 Outage probability comparison with effect of interference from PT; $P_{P T} / N_{0}=10 \mathrm{~dB}$

larger. This is because, when $I_{\text {th }} / N_{0}$ is larger, the instantaneous transmit power is no longer a function of $I_{\text {th }}$, rather it depends on $P$ as can be seen in (53). Since $P$ is set as a constant, the rate also remains as a constant in this region.

In the next set of figures, we consider the effect of primary interference on the secondary network.
Figures 11, 12 and 13 show the outage probability results under the influence of primary interference. The results establish that the outage probability increases in the presence of PT. In CogFDR/HDR networks, the outage floor which is seen to occur in the high transmit power region, happens for lower values of $P / N_{0}$ when the effect of PT is

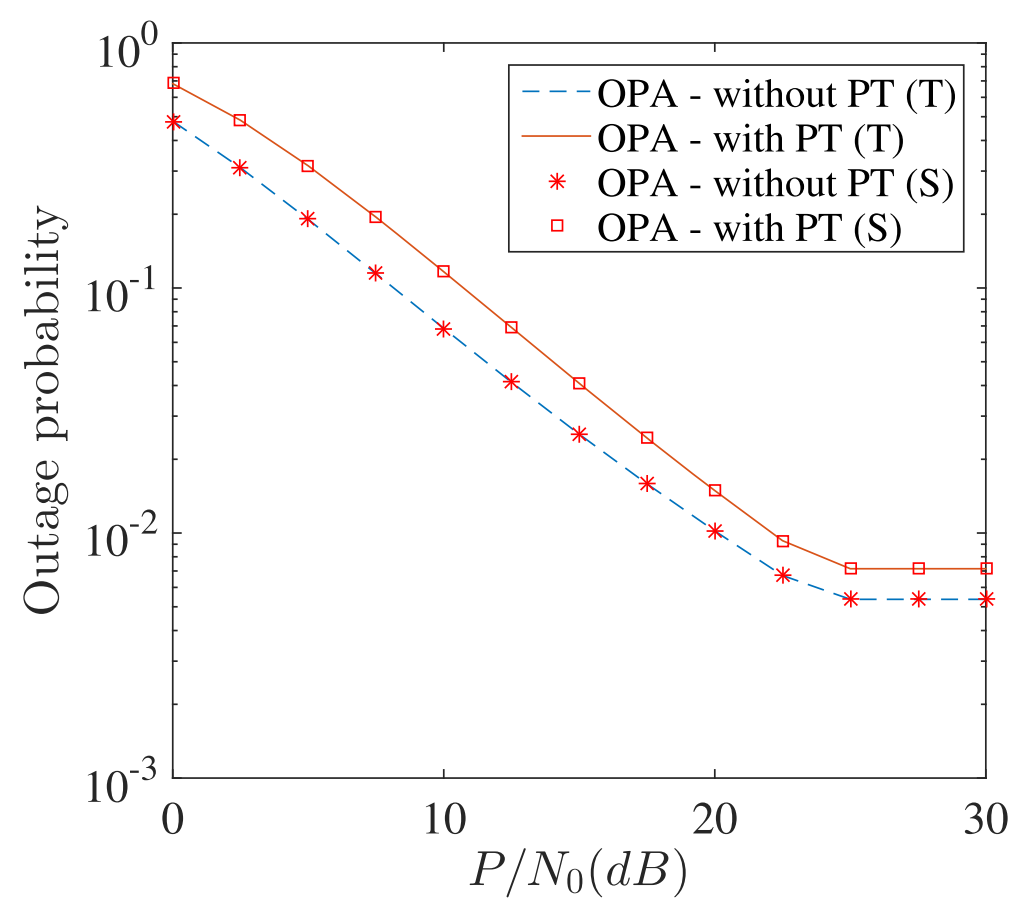

Fig. 12 Outage probability comparison with and without interference from PT; $P_{P T} / N_{0}=10 \mathrm{~dB}$ 


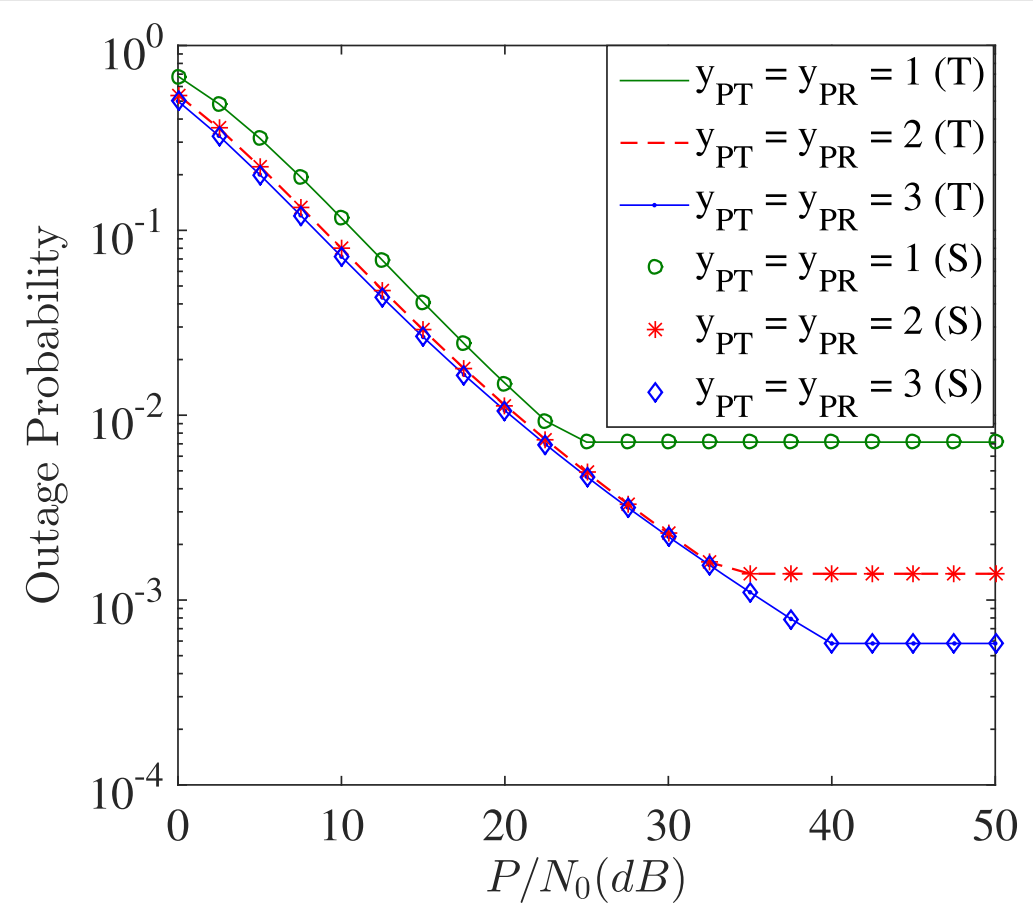

Fig. 13 Outage probability comparison for various $y$-coordinate locations of PT-PR pair; $P_{P T} / N_{0}=10 \mathrm{~dB}$

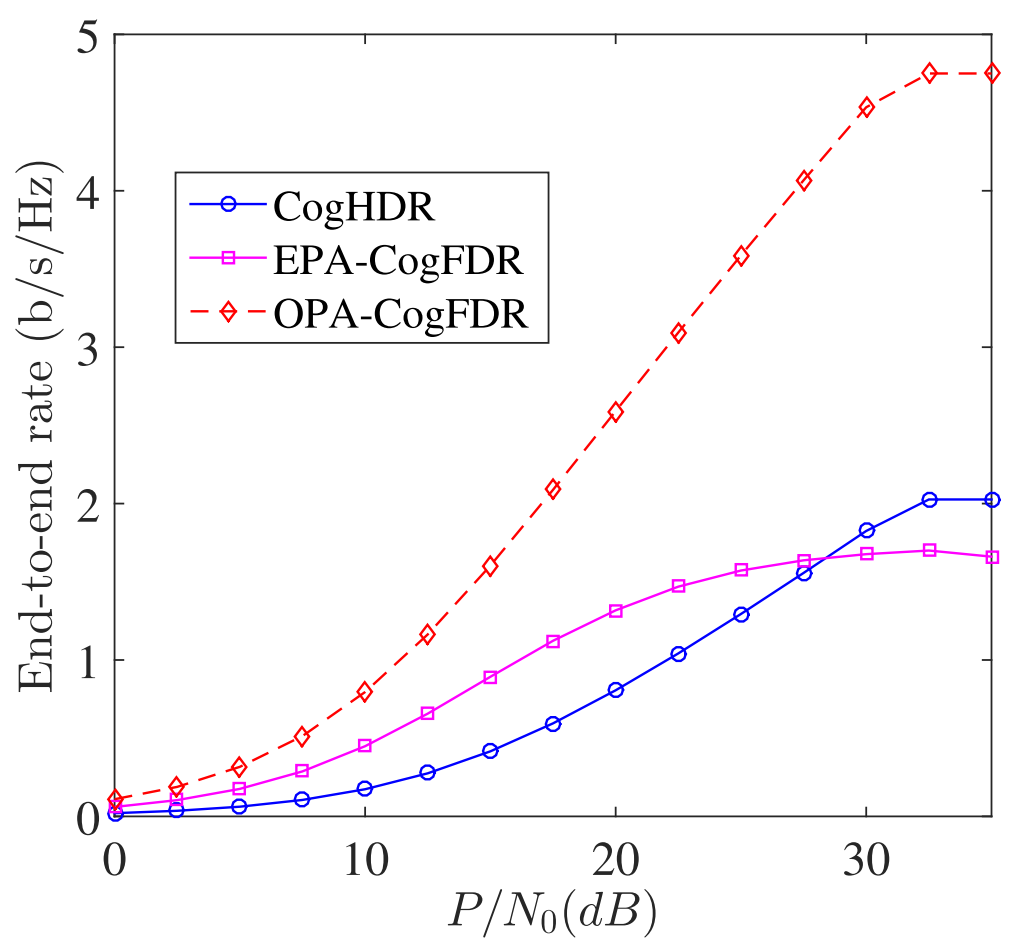

Fig. 14 End-to-end rate instantaneous comparison with effect of interference from PT; $P_{P T} / N_{0}=10 \mathrm{~dB}$ 


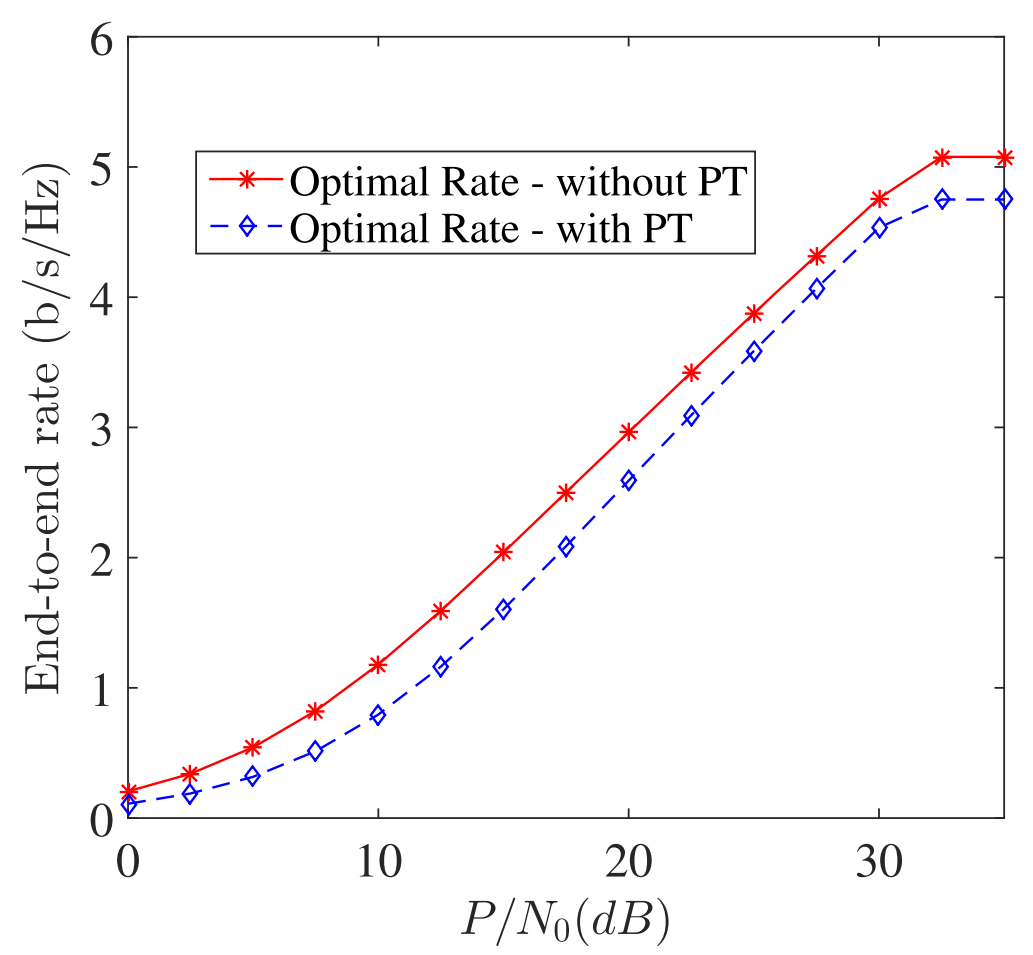

Fig. 15 End-to-end instantaneous rate comparison with and without interference from PT; $P_{P T} / N_{0}=10 \mathrm{~dB}$

considered. Further, the results show that the outage probability increases by $46 \%$ for $P / N_{0}=20 \mathrm{~dB}$ and $P_{P T} / N_{0}=$ $10 \mathrm{~dB}$. Figure 13 shows the impact of $y$-coordinate location of PT and PR on outage of secondary network. Notice that the outage probability decreases by $40 \%$ as the $P T-P R$ pair moves from $y_{P T}=y_{P R}=1$ to $y_{P T}=y_{P R}=3$.

Figures 14 and 15 show the end-to-end instantaneous rate taking the interference from PT into consideration. The instantaneous value of channel gain from PT to the secondary nodes $F_{1}, F_{2}$, and $F_{3}$ is given as follows:

$$
H_{P T}=\left[\begin{array}{lll}
0.1807 & 0.0825 & 0.0195
\end{array}\right]
$$

where the elements denote $\left|h_{P T, j}\right|^{2}, j=\{1,2,3\}$, which are also generated from exponential random variables. Figure 14 shows the comparison of optimal end-to-end instantaneous rate of CogFDR against that of CogHDR and CogFDR-EPA. The end-to-end rate outperforms all the other cases, under OPA. Figure 15 shows that the endto-end rate obtained under the effect of PT is $14 \%$ less than that obtained without PT (at $P / N_{0}=20 \mathrm{~dB}$ and $\left.P_{P T} / N_{0}=10 \mathrm{~dB}\right)$.

Overall, the results demonstrate that transmit power optimization can cause notable enhancement in the instantaneous rate and outage probability performance of CogFDR systems with respect to CogFDR-EPA and CogHDR systems. However, the interference from PT reduces the instantaneous rate and increases the outage probability, and the proposed OPA scheme ensures that even under the effect of interference from PT, we obtain good outage and rate performance. The power allocation schemes can be implemented in a centralized manner. The secondary nodes must have the ICSI/SCSI of the secondary transmission links, the links from $\mathrm{PT}$ to $F_{j}$ and the links from $F_{j}$ to PR. The ICSI can be obtained with the help of ICSI feedback or pilot-aided channel estimation. Each node, after learning the channel to every other node, have to communicate the acquired information to the centralized controller, which has to run the algorithm and compute the OPA vector and distribute the power values among the nodes.

\section{Conclusion}

In this work, we have developed analytical models for finding the outage probability of the secondary user in an underlay multi-hop cognitive FDR system considering the impact of residual self-interference (RSI) due to full-duplex operation, inter-relay interference (IRI) arising due to frequency re-use, and interference induced by primary transmitter on the secondary nodes. To improve the outage probability and the end-to-end rate performance of the secondary user, optimal selection of powers for the secondary nodes were considered. We formulated an optimization problem in order to determine the optimal power allocation vector for the secondary nodes that 
would minimize the outage probability of the secondary network subject to constraints on total power available in the secondary network and also the tolerable interference power at the primary receiver. In order to maximize the end-to-end rate, a power allocation method based on SINR balancing was formulated. Monte Carlo simulations were carried out to substantiate the analytical results. The transmission rate and outage probability of the multi-hop CogFDR network improved significantly when transmit power optimization was employed, in comparison to equal power allocation method.

\section{Appendix A: Derivation of (9):}

$$
\begin{aligned}
& \operatorname{Pr}\left(\Gamma_{j, \mathrm{FDR}}^{(0)} \geq \Gamma_{T}\right)=\operatorname{Pr}\left(X_{j-1, j} \geq\left(1+Z_{j}\right) \Gamma_{T}\right) \\
& =\int_{0}^{\infty} \int_{(1+z) \Gamma_{T}}^{\infty} f_{X_{j-1, j}}(x) f_{Z_{j}}(z) \mathrm{dxdz} \\
& =\int_{0}^{\infty} \int_{(1+z) \Gamma_{T}}^{\infty} \frac{1}{\beta_{j-1, j}} \exp \left(-\frac{x}{\beta_{j-1, j}}\right) \\
& d x \frac{1}{\left(\beta_{j+1, j}-\beta j, j\right)}\left(\exp \left(-\frac{z}{\beta_{j+1, j}}\right)-\exp \left(-\frac{z}{\beta_{j, j}}\right)\right) d z \\
& =\int_{0}^{\infty} \exp \left(-\frac{(1+z) \Gamma_{T}}{\beta_{j-1, j}}\right) \frac{1}{\left(\beta_{j+1, j}-\beta j, j\right)} \\
& \left(\exp \left(-\frac{z}{\beta_{j+1, j}}\right)-\exp \left(-\frac{z}{\beta_{j, j}}\right)\right) d z \\
& =\frac{\exp \left(-\frac{\Gamma_{T}}{\beta_{j-1, j}}\right)}{\left(\beta_{j+1, j}-\beta j, j\right)} \int_{0}^{\infty}\left(\exp \left(-z\left(\frac{\Gamma_{T}}{\beta_{j-1, j}}+\frac{1}{\beta_{j+1, j}}\right)\right)\right. \\
& -\exp \left(-z\left(\frac{\Gamma_{T}}{\beta_{j-1, j}}+\frac{1}{\beta_{j, j}}\right)\right) d z \\
& =\frac{\exp \left(-\frac{\Gamma_{T}}{\beta_{j-1, j}}\right)}{\left(\beta_{j+1, j}-\beta j, j\right)}\left[\frac{1}{\left(\frac{\Gamma_{T}}{\beta_{j-1, j}}+\frac{1}{\beta_{j+1, j}}\right)}-\frac{1}{\left(\frac{\Gamma_{T}}{\beta_{j-1, j}}+\frac{1}{\beta_{j, j}}\right)}\right] \\
& =\frac{\exp \left(-\frac{\Gamma_{T}}{\beta_{j-1, j}}\right)}{\left(1+\frac{\Gamma_{T} \beta_{j+1, j}}{\beta_{j-1, j}}\right)\left(1+\frac{\Gamma_{T} \beta_{j, j}}{\beta_{j-1, j}}\right)}
\end{aligned}
$$

\section{Appendix B: Derivation of (23a):}

Let us define the variable $W_{j}=X_{j, j}+X_{j+1, j}+X_{P T, j}$. Using Welch-Satterthwaite approximation [44] for the PDF of the sum of exponential random variables, we have,

$$
f_{W_{j}}(w)=\frac{\beta^{m}}{\Gamma(m)} w^{m-1} e^{-w / \beta}
$$

where $m$ and $\beta$ are given by,

$$
\begin{gathered}
m=\frac{\left(\beta_{j, j}+\beta_{j+1, j}+\beta_{P T, j}\right)^{2}}{\beta_{j, j}^{2}+\beta_{j+1, j}^{2}+\beta_{P T, j}^{2}} \\
\beta=\frac{\beta_{j, j}^{2}+\beta_{j+1, j}^{2}+\beta_{P T, j}^{2}}{\beta_{j, j}+\beta_{j+1, j}+\beta_{P T, j}}
\end{gathered}
$$

$$
\begin{aligned}
\operatorname{Pr}\left(\Gamma_{j, \mathrm{FDR}}^{(1)} \geq \Gamma_{T}\right) & =\operatorname{Pr}\left(\frac{X_{j-1, j}}{1+W_{j}} \geq \Gamma_{T}\right) \\
& =\int_{0}^{\infty} \operatorname{Pr}\left(X_{j-1, j} \geq \Gamma_{T}(1+w)\right) f_{W_{j}}(w) d z \\
& =\int_{0}^{\infty} e^{-\frac{\Gamma_{T}(1+w)}{\beta_{j-1, j}}} \frac{\beta^{-m}}{\Gamma(m)} w^{m-1} e^{-w / \beta} d w \\
& =e^{-\frac{\Gamma_{T}}{\beta_{j-1, j}}} \beta^{-m}\left(\frac{\Gamma_{T}}{\beta_{j-1, j}}+\frac{1}{\beta}\right)^{-m} \\
& =e^{-\frac{\Gamma_{T}}{\beta_{j-1, j}}}\left(1+\frac{\Gamma_{T} \beta}{\beta_{j-1, j}}\right)^{-m}
\end{aligned}
$$

\section{Appendix C: Derivation of (27):}

To find $\operatorname{Pr}\left(\Gamma_{j, \mathrm{HDR}}^{(1)} \geq \gamma_{T}\right)$, we make use of (26). Thus, we have the following equations:

$$
\begin{aligned}
\operatorname{Pr}\left(\Gamma_{j, \mathrm{HDR}}^{(1)} \geq \gamma_{T}\right) & =\operatorname{Pr}\left(X_{j-1, j} \geq \gamma_{T}\left(1+X_{P T, j}\right)\right) \\
& =\int_{0}^{\infty} e^{-\frac{\gamma_{T}(1+x)}{\beta_{j-1, j}}} f_{X_{P T, j}}(x) d x \\
& =\int_{0}^{\infty} e^{-\frac{\gamma_{T}(1+x)}{\beta_{j-1, j}}} \frac{1}{\beta_{P T, j}} e^{-\frac{x}{\beta_{P T, j}}} d x \\
& =\frac{e^{-\frac{\gamma_{T}}{\beta_{j-1, j}}}}{\beta_{P T, j}} \int_{0}^{\infty} e^{-x\left(\frac{\gamma_{T}}{\beta_{j-1, j}}+\frac{1}{\beta_{P T, j}}\right)} d x \\
& =\frac{e^{-\frac{\gamma_{T}}{\beta_{j-1, j}}}}{\left(1+\frac{\gamma_{T} \beta_{P T, j}}{\beta_{j-1, j}}\right)}
\end{aligned}
$$

Now $P_{\text {out,HDR }}^{(1)}$ can be determined using (58).

\section{Abbreviations}

AF: Amplify-and-forward; CogFDR: Cognitive-based FDR; CogHDR: Cognitive-based HDR; CR: Cognitive radio; CRN: Cognitive relay network; DF: Decode-and-forward; EPA: Equal power allocation on average; OPA: Optima power allocation; FD: Full-duplex; FDR: Full-duplex relaying; HD: Half-duplex; HDR: Half-duplex relaying; ICSI: Instantaneous channel state information; IRI: Inter-relay interference; PR: Primary receiver; PT: Primary transmitter; RSI: Residual self-interference; SCSI: Statistical channel state information; SR; Secondary receiver; ST: Secondary transmitter

\section{Acknowledgements}

Not applicable.

Funding

Not applicable.

\section{Availability of data and materials} Not applicable.

\section{Authors' information}

Not applicable.

\section{Authors' contributions}

All authors have contributed to this research work. Both authors have read and approved the final manuscript.

\section{Competing interests}

The authors declare that they have no competing interests.

\section{Publisher's Note}

Springer Nature remains neutral with regard to jurisdictional claims in published maps and institutional affiliations. 
Received: 17 August 2018 Accepted: 12 November 2018 Published online: 06 December 2018

\section{References}

1. S. Haykin, Cognitive radio: brain-empowered wireless communications IEEE J. Sel. Areas Commun. 23(2), 201-220 (2005)

2. B. Wang, K. R. Liu, Advances in cognitive radio networks: a survey. IEEE J. Sel. Top. Signal Process. 5(1), 5-23 (2011)

3. K. B. Letaief, W. Zhang, Cooperative communications for cognitive radio networks. Proc. IEEE. 97(5), 878-893 (2009)

4. M. Duarte, C. Dick, A. Sabharwal, Experiment-driven characterization of full-duplex wireless systems. IEEE Trans. Wirel. Commun. 11(12), 4296-4307 (2012)

5. Z. Zhang, X. Chai, K. Long, A. V. Vasilakos, L. Hanzo, Full-duplex techniques for $5 \mathrm{G}$ networks: self-interference cancellation, protocol design, and relay selection. IEEE Commun. Mag. 53(5), 128-137 (2015)

6. E. Everett, D. Dash, C. Dick, A. Sabharwal, in 2011 IEEE 49th Annual Allerton Conference on Communication, Control, and Computing. Self-interference cancellation in multi-hop full-duplex networks via structured signaling (IEEE, Allerton, 2011), pp. 1619-1626

7. T. Kwon, S. Lim, S. Choi, D. Hong, Optimal duplex mode for df relay in terms of the outage probability. IEEE Trans. Veh. Technol. 59(7), 3628-3634 (2010)

8. G. Liu, F. R. Yu, H. Ji, V. C. Leung, X. Li, In-band full-duplex relaying: a survey, research issues and challenges. IEEE Commun. Surv. Tutor. 17(2), 500-524 (2015)

9. T. Riihonen, S. Werner, R. Wichman, in 2009 IEEE Wireless Communications and Networking Conference. Comparison of full-duplex and half-duplex modes with a fixed amplify-and-forward relay (IEEE, Budapest, 2009), pp. 1-5

10. Y. Liao, L. Song, Z. Han, Y. Li, Full-duplex cognitive radio: a new design paradigm for enhancing spectrum usage. IEEE Commun. Mag. 53(5), 138-145 (2015)

11. S. I. Hussain, M. M. Abdallah, M.-S. Alouini, K. Qaraqe, M. Hasna, Relay selection in underlay cognitive networks with fixed transmission power nodes. Trans. Emerg. Telecommun. Technol. 24(7-8), 734-747 (2013)

12. W. Lin, Y. Wang, F. Y. Li, in 2015 IEEE 81st Vehicular Technology Conference (VTC Spring). A multi-phase decode-and-forward transmission protocol in cognitive relay networks: outage analysis and relay power allocation (IEEE, Glasgow, 2015), pp. 1-5

13. J. Zhu, J. Huang, W. Zhang, in International Conference on Wireless Communications and Signal Processing (WCSP). Optimal one-dimensional relay placement in cognitive radio networks, vol. 2010 (IEEE, Suzhou, 2010), pp. 1-6

14. T.-T. Tran, V. N. Q. Bao, V. D. Thanh, T. Q. Duong, in 2013 International Conference on Computing, Management and Telecommunications (ComManTel). Performance analysis and optimal relay position of cognitive spectrum-sharing dual-hop decode-and-forward networks (IEEE, Ho Chi Minh City, 2013), pp. 269-273

15. Z. Shu, W. Chen, in 2010 IEEE International Conference on Wireless Communications, Networking and Information Security (WCNIS). Optimal power allocation in cognitive relay networks under different power constraints (IEEE, Beijing, 2010), pp. 647-652

16. X. Ge, Y. Sun, H. Gharavi, J. Thompson, Joint optimization of computation and communication power in multi-user massive MIMO systems. IEEE Trans. Wirel. Commun. 17(6), 4051-4063 (2018)

17. Q. Li, S. Feng, A. Pandharipande, X. Ge, Q. Ni, J. Zhang, Wireless-powered cooperative multi-relay systems with relay selection. IEEE Access. $\mathbf{5}$, 19058-19071 (2017)

18. Q. Li, S. Feng, X. Ge, G. Mao, L. Hanzo, On the performance of full-duplex multi-relay channels with DF relays. IEEE Trans. Veh. Technol. 66(10), 9550-9554 (2017)

19. Q. Li, M. Yu, A. Pandharipande, X. Ge, J. Zhang, J. Zhang, Performance of virtual full-duplex relaying on cooperative multi-path relay channels. IEEE Trans. Wirel. Commun. 15(5), 3628-3642 (2016)

20. S. B. Mafra, H. Alves, D. B. Costa, R. D. Souza, E. M. G. Fernandez, M Latva-aho, On the performance of cognitive full-duplex relaying under spectrum sharing constraints. EURASIP J. Wirel. Commun. Netw. 2015(1), 1-13 (2015)

21. N. Li, Y. Li, T. Wang, M. Peng, W. Wang, in 2015 IEEE 26th Annual International Symposium on Personal, Indoor, and Mobile Radio Communications (PIMRC). Full-duplex based spectrum sharing in cognitive two-way relay networks (IEEE, Hong Kong, 2015), pp. 997-1001
22. H. Chen, S. Tan, F. Zhao, Outage performance of relay-assisted transmissions in cognitive full-duplex relay networks. EURASIP J. Wirel. Commun. Netw. 2015(1), 1-11 (2015)

23. Y. Shi, L. Zhang, Z. Chen, Y. Gong, G. Wu, in 2013 IEEE Globecom Workshops (GC Wkshps). Optimal power allocation for AF full-duplex relay in cognitive radio networks (IEEE, Atlanta, 2013), pp. 322-327

24. X.-T. Doan, N.-P. Nguyen, C. Yin, D. B. Da Costa, T. Q. Duong, Cognitive full-duplex relay networks under the peak interference power constraint of multiple primary users. EURASIP J. Wirel. Commun. Netw. 2017(1), $1-10(2017)$

25. L. T. Tan, L. Ying, D. W. Bliss, in 51st Annual Conference on Information Sciences and Systems (CISS). Power control and relay selection in full-duplex cognitive relay networks: coherent versus non-coherent scenarios (IEEE, Baltimore, 2017), pp. 1-6

26. H. Kim, S. Lim, H. Wang, D. Hong, Optimal power allocation and outage analysis for cognitive full-duplex relay systems. IEEE Trans. Wirel. Communun. 11(10), 3754-3765 (2012)

27. C. Yin, T. X. Doan, N.-P. Nguyen, T. Mai, L. D. Nguyen, in International Conference on Recent Advances in Signal Processing, Telecommunications \& Computing (SigTelCom). Outage probability of full-duplex cognitive relay networks with partial relay selection (IEEE, Da Nang, 2017), pp. 115-118

28. S. Rajkumar, J. S. Thiruvengadam, Outage analysis of OFDM based cognitive radio network with full-duplex relay selection. IET Signal Proc. 10(8), 865-872 (2016)

29. Y. Shi, L. Zhang, Z. Chen, Y. Gong, G. Wu, in 2013 IEEE Globecom Workshops (GC Wkshps). Optimal power allocation for AF full-duplex relay in cognitive radio networks (IEEE, Atlanta, 2013), pp. 322-327

30. M. A. Girnyk, M. Xiao, L. K. Rasmussen, in 2012 Swedish Communication Technologies Workshop (Swe-CTW). Power allocation for multi-hop decode-and-forward cognitive radio networks with line topology (IEEE, Lund, 2012), pp. 7-12

31. N. T. Van, T. T. Duy, T. Hanh, V. N. Q. Bao, in 2017 International Symposium on Antennas and Propagation (ISAP). Outage analysis of energy-harvesting based multihop cognitive relay networks with multiple primary receivers and multiple power beacons (IEEE, Phuket, 2017), pp. 1-2

32. S. Mondal, S. D. Roy, S. Kundu, Primary behaviour-based energy harvesting multihop cognitive radio network. IET Commun. 11(16), 2466-2475 (2017)

33. H. K. Boddapati, S. Prakriya, M. R. Bhatnagar, in 2016 IEEE 83rd Vehicular Technology Conference (VTC Spring). Outage analysis of cluster-based multi-hop cognitive radio networks (IEEE, Nanjing, 2016), pp. 1-5

34. S. Mondal, S. Dhar Roy, S. Kundu, Energy harvesting based multihop relaying in cognitive radio network. Wirel. Pers. Commun. 97(4), 6325-6342 (2017)

35. K. Ho-Van, Influence of channel information imperfection on outage probability of cooperative cognitive networks with partial relay selection. Wirel. Pers. Commun. 94(4), 3285-3302 (2017)

36. A. Goldsmith, Wireless communications. (Cambridge university press, 2005)

37. M. Xia, S. Aissa, Cooperative AF relaying in spectrum-sharing systems: performance analysis under average interference power constraints and Nakagami-m fading. IEEE Trans. Commun. 60(6), 1523-1533 (2012)

38. S. P. Boyd, L. Vandenberghe, Convex optimization (pdf). (Np: Cambridge UP, 2004)

39. M. Grant, S. Boyd, CVX: Matlab software for disciplined convex programming (web page and software) (2009). http://stanford.edu/boyd/cvx

40. M. Sikora, J. N. Laneman, M. Haenggi, D. J. Costello, T. E. Fuja, Bandwidth-and power-efficient routing in linear wireless networks. IEEE Trans. Inf. Theory. 52(6), 2624-2633 (2006)

41. H. Jeong, J. H. Lee, in IEEE 68th Vehicular Technology Conference, 2008. VTC 2008-Fall. Adaptive relay selection for regenerative OFDMA relay networks with fairness constraints (IEEE, Calgary, 2008), pp. 1-5

42. D. Bharadia, G. Bansal, P. Kaligineedi, V. K. Bhargava, Relay and power allocation schemes for OFDM-based cognitive radio systems. IEEE Trans. Wirel. Commun. 10(9), 2812-2817 (2011)

43. R. J. Schilling, S. L. Harris, Applied numerical methods for engineers using MATLAB and C. (Brooks/cole, Pacific Grove, 2000)

44. F. E. Satterthwaite, An approximate distribution of estimates of variance components. Biom. Bull. 2(6), 110-114 (1946) 